OPEN ACCESS

Edited by: Gregory Light, University of California, San Diego, United States

Reviewed by:

Teresa Sanchez-Gutierrez, Universidad Internacional De La Rioja, Spain

Massimo Tusconi,

University of Cagliari, Italy

*Correspondence: Verner Knott

Verner.Knott@theroyal.ca

Specialty section: This article was submitted to Schizophrenia a section of the journal

Frontiers in Psychiatry

Received: 05 March 2020 Accepted: 01 June 2020

Published: 12 June 2020

Citation:

Knott V, Wright N, Shah D, Baddeley A, Bowers $H$, de la Salle $S$ and Labelle $A$ (2020) Change in the Neural Response to Auditory Deviance Following Cognitive Therapy for Hallucinations in Patients With Schizophrenia.

Front. Psychiatry 11:555 doi: 10.3389/fpsyt.2020.00555

\section{Change in the Neural Response to Auditory Deviance Following Cognitive Therapy for Hallucinations in Patients With Schizophrenia}

\author{
Verner Knott ${ }^{1,2,3 *}$, Nicola Wright ${ }^{4}$, Dhrasti Shah ${ }^{1}$, Ashley Baddeley ${ }^{2}$, Hayley Bowers ${ }^{4}$, \\ Sara de la Salle ${ }^{1,2}$ and Alain Labelle 3,4 \\ ${ }^{1}$ School of Psychology, University of Ottawa, Ottawa, ON, Canada, ${ }^{2}$ Clinical Neuroelectrophysiology and Cognitive \\ Research Laboratory, University of Ottawa Institute of Mental Health Research, Ottawa, ON, Canada, ${ }^{3}$ Department of \\ Psychiatry, University of Ottawa, Ottawa, ON, Canada, ${ }^{4}$ Schizophrenia Program, The Royal Ottawa Mental Health Centre, \\ Ottawa, ON, Canada, ${ }^{5}$ Department of Psychology, University of Guelph, Guelph, ON, Canada
}

Adjunctive psychotherapeutic approaches recommended for patients with schizophrenia (SZ) who are fully or partially resistant to pharmacotherapy have rarely utilized biomarkers to enhance the understanding of treatment-effective mechanisms. As SZ patients with persistent auditory verbal hallucinations (AVH) frequently evidence reduced neural responsiveness to external auditory stimulation, which may impact cognitive and functional outcomes, this study examined the effects of cognitive behavioral therapy for voices (CBTV) on clinical and AVH symptoms and the sensory processing of auditory deviants as measured with the electroencephalographically derived mismatch negativity (MMN) response. Twenty-four patients with $\mathrm{SZ}$ and $\mathrm{AVH}$ were randomly assigned to group CBTV treatment or a treatment as usual (TAU) condition. Patients in the group CBTV condition received treatment for 5 months while the matched control patients received TAU for the same period, followed by 5 months of group CBTV. Assessments were conducted at baseline and at the end of treatment. Although not showing consistent changes in the frequency of AVHs, CBTv (vs. TAU) improved patients' appraisal ( $p=$ 0.001) of and behavioral/emotional responses to $\mathrm{AVHs}$, and increased both MMN generation ( $p=0.001)$ and auditory cortex current density $(p=0.002)$ in response to tone pitch deviants. Improvements in AVH symptoms were correlated with change in pitch deviant MMN and current density in left primary auditory cortex. These findings of improved auditory information processing and symptom-response attributable to CBTV suggest potential clinical and functional benefits of psychotherapeutical approaches for patients with persistent AVHs.

Keywords: schizophrenia, auditory hallucinations, cognitive behavioral therapy, auditory cortex, auditory sensory processing, mismatch negativity 


\section{INTRODUCTION}

Auditory verbal hallucinations (AVHs), defined as perceptions or subjective experiences of "hearing voices" without corresponding external auditory stimulation, occur with a high frequency of up to $60 \%$ to $80 \%$ in patients with schizophrenia (SZ) (1). Reflecting a diverse phenomenological experience, AVHs can involve words, sentences, or conversations (with varied clarity, loudness, and spatial locations) spoken as commands, comments, insults, or encouragements by familiar or unfamiliar single and/or multiple voices (in first, second, or third person) (2).

Although the causes of AVHs are still unclear, improved understanding of the neural basis of AVHs has been forthcoming from functional magnetic resonance imaging (fMRI) studies which have shown elevated activation of brain regions associated with auditory stimulus processing, speech generation, and speech perception during the experience of active hallucinations (vs. silent rest) (3-6). Paradoxically, although in sensory cortices hyper-excitable neuronal states are typically associated with enhanced exogenous induced processes (7-10), AVHs have been associated with reduced neuronal activation of the auditory cortex in response to external auditory stimulation (11). These opposing findings in hallucinating patients of increased activation of the auditory cortex in the absence of external stimulation and reduced activation of the auditory cortex in response to externally presented speech and non-speech sounds have been interpreted as evidence for competition between internally generated and externally originating neural activity in the auditory cortex for the attentional resources of the hallucinating patient (11). Also evidenced in SZ patients who are prone to AVHs (vs. patients who have never hallucinated), diminished neural responsiveness to external auditory stimulation is believed to affect the functional cost of an auditory cortex that is thought to be tonically "tuned on" and "tuned in" to the internal channels broadcasting hallucinating stimuli, with the preferential endogenous processing of AVHs resulting in the "saturation" of neuronal resources and resulting in limited capacity for the exogenous processing of external auditory stimuli $(12,13)$.

Further evidence that the auditory cortex in hallucinating patients is overly sensitive to activation arising from internal processing, while being less responsive to external stimulation, comes from electrophysiological studies assessing cortical responsiveness to auditory stimuli with electroencephalographically (EEG)-derived event-related potential (ERP) components that have been shown to be generated in the auditory cortex and have been extensively used to document profound early auditory information processing (EAIP) deficits in SZ (14). Patients with SZ have been found to be impaired with respect to two aspects of EAIP: inhibiting intrinsic responses to redundant stimuli (to prevent sensory overload), and facilitating/ detecting potentially salient stimuli (for extended higher-order processing and response) (15). These elementary pre-attentive auditory input deficits in SZ are reflected in two candidate ERP endophenotypes, one of which includes P50 sensory gating as a measure of inhibitory failure. This inhibitory deficit is indexed in SZ both by minimal suppression of P50 (an early central-maximum positive scalp component elicited at $\sim 50 \mathrm{~ms}$ in response to the second stimulus $\left[S_{2}\right]$ of click pairs $\left.\left[S_{1}-S_{2}\right]\right)$, and by a diminished $S_{1}$ P50 amplitude (16). A second ERP endophenotype of EAIP dysfunction in SZ, mismatch negativity (MMN), is a frontal maximum negative scalp component at $\sim 150$ to $200 \mathrm{~ms}$ which indexes automatic acoustic deviance detection and, in SZ, exhibits a reduced amplitude in response to changes in physical or abstract features in auditory oddball paradigms (17).

Although both of these ERP-indexed elementary sensory processes (auditory gating and auditory charge detection) have been consistently shown to be abnormal in SZ, our findings indicated a significant worsening of these brain sensory functions in patients who hallucinate (trait positive) as: (1) increasing negative affective content of AVHs was inversely related with $S_{1}$ P50 amplitude (18); (2) SZ hallucinators (vs. non-hallucinators) exhibited smaller MMNs to changes in pure tone stimuli (19), with MMN reduction being more evident with increasing trait ratings of hallucinatory activity (20); and (3) SZ hallucinators (vs. healthy controls) showed smaller MMNs to pure tone and speech deviant stimuli (21). Furthermore, in SZ patients who are prone to hallucinate, we observed diminished involuntary attentional orienting to speech stimuli (evidenced by a reduction in a later $[\sim 300 \mathrm{~ms}]$ frontocentral positive $\left[\mathrm{P} 3_{\mathrm{a}}\right]$ scalp component), suggesting an impairment in the ability of human speech deviations to capture attention (22). Together with findings of reduced amplitude of the N1 component of the auditory ERP during hallucinating states (23), observed ERP deficits in sensory registration (N1), sensory inhibition (P50), sensory discrimination $(\mathrm{MMN})$, and stimulus selection $\left(\mathrm{P}_{\mathrm{a}}\right)$ within the auditory modality are consistent with the "saturation" hypothesis of AVHs. The resulting competitive outcome favoring resource allocation to the processing of internal auditory signals may in part explain the profound behavioral performance deficits of SZ patients during auditory discrimination tasks (24).

AVHs are associated with high levels of distress likely related to idiosyncratic beliefs or cognitive appraisals involving control, power, voice identity, authority, and consequences of not complying with the voices (25-27). Despite adequate dosages of antipsychotic drugs, AVHs are drug resistant in 25\% of SZ patients, and become chronic, causing an impaired quality of life (28) and diminished cognitive capacity, with the latter playing a key role in functional outcome (29). Cognitive behavioral therapy (CBT) has been suggested as a complement to pharmacotherapy for targeting psychosis in treatment resistant cases (30-32). Reviews (33-37) and multiple meta-analyses (38, $39,40-42)$ on the effectiveness of specialized cognitive behavioral therapy for psychosis (CBTp), developed, and recommended as an adjunctive treatment for decreasing distress in patients with persistent AVHs $(43,44)$, found a modest but significant positive impact on positive symptoms, negative symptoms, and general psychopathology. The proposed mechanism of change resulting from CBTv is through changes in beliefs about voices as well as enhancing coping skills (33).

In contrast to CBTp, which is aimed at a broad array of symptoms, administering tailored therapies for specific 
symptoms and using a recommended symptoms specific approach such as CBT for voices (CBTv) $(34,40)$ has shown effectiveness in individual and group sessions. In three randomized controlled group CBTv trials, improvements have been found not only in positive and general symptoms, but also in self-esteem, effective coping strategies and social functioning, as well as reductions in voice frequency and perceived voice power $(35,45-49)$.

\section{The Present Study}

The ultimate goal of CBTv is to help patients cope with auditory hallucinations, which would presumably translate into improved external auditory information processing, and to improved functioning. The primary aim of this pilot study was to examine change in the neuronal response to auditory stimulation following an integrated group CBTv trial which would incorporate the use of both acceptance and commitment therapy (ACT) to modify painful and stressful thoughts and emotions arising from voices (50-52), and attentional training (ATT) to reduce the attentional capture by emotionally salient voices $(53,54)$. Effective cognitive strategies that are able to reduce $\mathrm{AVH}$ saturation of sound perception neurocircuitry may free up resources for external auditory processing in limited capacity auditory cortical networks. At the sensory processing level, the MMN may be an ideal probe for indexing treatment associated with functional changes in the auditory cortex as: it can be rapidly assessed and it is highly stable over time (test-retest ranging from 0.60 to 0.80 ) $(55,56)$; it is an automatic sensory process that is relatively free of attentional and motivational confounds that influence effortdemanding, higher order cognitive operations (57); and finally, because MMN has strong external face validity (in that it is positively related to performance in behavioral tasks of sound discrimination) $(58,59)$, and its impairment in SZ is positively correlated with cognitive (memory) (60) and executive functioning deficits $(61,62)$, social skills acquisition (63), and global daily functioning (64-67).

In addition to our study's primary objective of using MMN to index CBTv-induced changes in neural correlates of auditory discrimination of pure tone deviants, a complimentary objective was to conduct a regions of interest (ROI) analysis on devianceelicited source localized activity in bilateral primary (pAC) and secondary auditory cortices ( $\mathrm{AC}$ ), putative regions implicated in AVHs, and the main cortical areas of MMN generation (68-76). Hypothetically, although we do not necessarily expect changes in AVH topography (i.e., frequency and quality of voices), within the "saturation" model we generally predicted that CBTv, in reducing resource-demanding processing of internal (voices) stimulation, will allow for increased processing of external auditory stimulation. At the neural level, we specifically hypothesized that increased exogenous processing following CBTv will be evidenced by greater MMN responses to auditory deviants, and by greater deviant-elicited activation in the primary auditory cortex (pAC), and specifically the left pAC as this is the main brain region in SZ hallucinators that exhibits both increased activation in the absence of an external stimulus and decreased activation in the presence of an external auditory stimulus (11). CBTv-induced changes in symptoms were expected to correlate with changes in deviance elicited MMN and auditory cortex responses. MMN changes with CBTv were also predicted to be related to response changes in the auditory cortex.

\section{METHODS}

\section{Study Participants}

The study was approved by the Research Ethics Board of the Royal Ottawa Mental Health Centre and the University of Ottawa. The study recruited twenty-five (10 women, 15 men) individuals with schizophrenia (SZ: $M=45.95$ years, $S D=12.60$ ) from the Outpatient Schizophrenia Program of the Royal Ottawa Mental Health Centre, all of which were diagnosed by trained psychiatrists using the Structured Clinical Interview DSM-IV-TR (SCID-I) (77). Patients included in the study: (i) were between the ages of 18 and 60 years; (ii) reported a consistent history of auditory verbal hallucinations over the course of their illness; (iii) exhibited a score of 3 or greater (reflecting mild or greater auditory/verbal hallucinatory experience) on the hallucination item of the Positive and Negative Syndrome Scale (PANSS) (78), and a score less than 65 on the total PANSS score (to screen out individuals with severe level of symptoms and severe impaired functioning that would impact their ability to participate in group CBTv); (iv) reported no history of neurological conditions or head injury; (v) were clinically stable, as indicated by no significant changes in symptoms or medication, for at least the 3-month period prior to testing; (vi) were being treated only with one of the atypical antipsychotics as their primary medication; (vii) were willing to participate in 5 to 6 months of CBTv in addition to their usual treatment; and (viii) displayed normal hearing (threshold $<30$ $\mathrm{dB}$ SPL) as assessed by audiometric testing.

\section{Treatment Design}

Following a parallel group design, 14 (8 males) of the 25 patients were randomly assigned to receive CBTv for 5 months in addition to their usual treatment (CBTv group) and eleven (7 males) were randomly assigned to continue their treatment as usual (TAU group). The recruitment and creation of groups involved: (i) a patient referral through hospital psychiatrist to the study team; (ii) the introduction of the study requirements and involvement by the study team and consent from participants; (iii) completion of screening session to ensure patients met the study requirements; and (iv) random assignment to treatment groups. In the CBTv group, patients received CBTv for 5 to 6 months, while patients in the TAU were followed for the same time period. Following completion, TAU patients then completed 5 months of CBTv treatment (Figure 1). The two laboratory test sessions, one at baseline and one at follow-up, included electrophysiological recordings, assessment of psychiatric symptoms, and completion of questionnaires relating to AVHs.

Of the fourteen patients assigned to the CBTv condition, thirteen patients ( 8 males) completed all assessments at baseline 


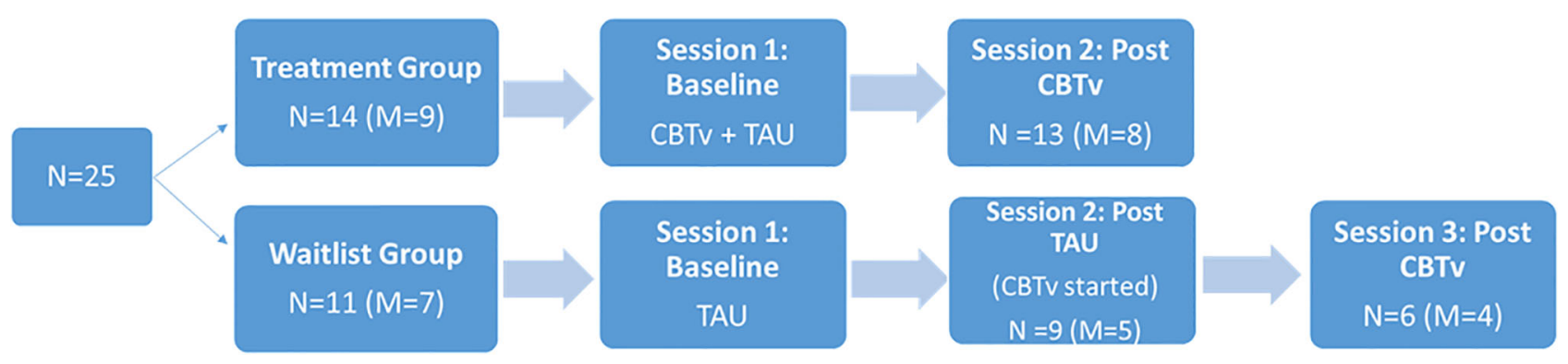

FIGURE 1 | Flow chart of treatment design.

and follow-up and provided usable EEG data. Of the eleven patients recruited in TAU group, nine ( 5 males) completed all assessments at baseline and follow-up and provided usable EEG data. Of the nine patients who completed the TAU group, six completed all assessment at baseline, follow-up, and post-CBTv, and provided usable EEG data. All patients who completed the study continued with their regular medication and psychosocial interventions throughout the study period. The main reasons for attrition or exclusions from the study were: (i) consent withdrawal; (ii) incomplete or unusable EEG data at one or both time points (e.g. noisy EEG data, less than 40 clean EEG epochs per deviant stimulus, and missing EEG channels; (iv) medication change; and (v) onset of medical illness.

\section{CBTv Protocol}

Consistent with the NICE (77) and PORT (78) guidelines, group CBTv was delivered using a manualized approach, where prescribed goals and techniques to be used during treatment sessions are outlined and followed throughout treatment. The treatment was implemented by one expert CBTv therapist (N.W.), following a session-by-session treatment manual. Conducted in eighteen planned sessions over 5 months, and facilitated by highly trained group leaders, the CBTv intervention incorporated CBT strategies for positive symptoms, and ATT as well as ACT within a CBT framework. The 18 session group CBTv was administered on a weekly basis for 5 months (during the last 2 months, sessions were spread out to every two weeks). Each CBTv group had approximately nine participants and each participant had a copy of the participant manual, which included all homework/practice assignments. Adherence to the CBTv protocol across the groups was assessed by adherence to the treatment manual and measured by the Cognitive Therapy Scale for Psychosis (CTS-Psy) (79).

\section{Symptom Assessment}

Patients in the CBTv group were assessed independently at two test sessions: at baseline, and at follow-up at the end of CBTv (5 months after baseline). The TAU group was assessed at three test sessions: baseline, at follow-up at the end of waitlist period (5 months after baseline) and at the end of CBTv (10 months after baseline). The following clinical outcome measures were implemented:
Positive and Negative Syndrome Scale (78). The Structured Clinical Interview for the PANSS is a 30-item rating scale designed to measure the presence and severity of psychopathology in patients with SZ, schizoaffective disorder, and other psychological disorders. The PANSS was completed by a trained clinician following a semistructured interview format and using available clinical information. The clinician was blind to the group assignments. Each item was rated by the clinician on a Likert scale ranging from 1 (not present) to 7 (extremely severe). Three subscales scored were derived: Positive Symptoms scores (possible range of scored: 9-49); Negative Symptoms Scores (possible range of scores: 7-49); and General Symptoms Scores (possible range of scores: 16-112).

The Psychotic Symptom Rating Scales (PSYRATS) (80). The PSYRATS includes two scales designed to measure the severity of a number of dimensions of auditory hallucinations and delusions. Only the Auditory Hallucinations subscale was administered to the patients, which includes an 11-item scale that assesses dimensions of auditory hallucinations. The items include frequency, duration, location, loudness, amount and intensity of distress, amount and intensity of negative content, disruption, controllability, and number of voices. Symptoms scores are rated on a 5-point ordinal scale (0-4). Items are summarized for a total score, and higher scores reflect more severe auditory hallucinations.

Beliefs About Voices Questionnaire-Revised (BAVQ-R) (81). The BAVQ-R is a 35 -item self-report questionnaire that measures perceptions about, and emotional and behavioral response to auditory verbal hallucinations. The items are rated on a 4-point scale ranging from 0 (disagree) to 3 (strongly agree). The questionnaire consists of five subscales measuring different meanings given to the voices: omnipotence with six items (e.g., "My voice is very powerful"), malevolence with six items (e.g., "My voice is persecuting me for no good reason"), resistance with nine items (four items for emotion: e.g., "My voice frightens me" and five items for behavior: e.g., "When I hear my voice usually I tell it to leave me alone"), benevolence with six items (e.g., "My voice wants to help me") and engagement with eight items (four for emotion: e.g., "My voice makes me feel calm" and four for behavior: e.g., "I seek the advice of my voice").

Voices Acceptance and Action Scale (VAAS) (82). The VAAS is a 31-item self-report questionnaire that measures acceptancebased beliefs (defined as a willingness on the part of the voice 
hearer to have voices in his or her life coupled with an effective, non-avoidant disengagement from them) and action-based beliefs (defined as behaviors that are self-directed rather than being a reaction to the voices). Both the 16 acceptance-based items (e.g., "My voices are just one part of my life") and the 15 action-based items (e.g., "My voices stop me from doing things I want to do") are scored on a 5-point scale: strongly disagree, disagree, unsure or neutral, agree, or strongly disagree.

Choice of Outcome in CBT for Psychoses (CHOICE) (83). This outcome measure was developed to be sufficiently generic to apply across different CBTp approaches and models, but sensitive enough to capture change. It consisted of a twodimensional 24-item self-report questionnaire, which provides measures for severity and satisfaction across a range of problems/ difficulties (e.g., "ways of dealing with distressing experiences [e.g., beliefs, thoughts, and voices]," "the ability to approach problems in a variety of ways").

\section{Auditory Paradigm}

ERP test sessions occurred in the morning (8-11 a.m.) following overnight abstinence of drugs, alcohol, caffeine and food. During the auditory stimulation, participants sat upright and viewed a silent video (The Blue Planet by BBC, 2001). In the optimal MMN paradigm (84), which was designed to elicit MMN responses to 5 separate auditory deviants, auditory tonal stimuli of $70 \mathrm{~dB}$ sound pressure level (SPL) were presented binaurally through headphones and consisted of standard $(\mathrm{p}=0.5)$ stimuli (composed of three sinusoidal partials of 500 , $1000,1500 \mathrm{~Hz}, 75 \mathrm{~ms}$ duration) that were randomly intermixed with deviant $(\mathrm{p}=0.5)$ stimuli. Stimulus onset asynchrony (SOA) was fixed at $500 \mathrm{~ms}$. The deviant tones differed from the standard tones in terms of pitch, duration, intensity, perceived location of sound origin, or contained a silent gap in the middle of the tone (i.e. gap deviants). The duration deviant was only $25 \mathrm{~ms}$ in duration (instead of $75 \mathrm{~ms}$ ). Half of the pitch deviants were $10 \%$ lower (composed of 450, 900, and $1350 \mathrm{~Hz}$ partials) and the other half were $10 \%$ higher (composed of 550, 110, $1650 \mathrm{~Hz}$ partials). Half of the intensity variants were at $80 \mathrm{~dB}$ and the other half at $60 \mathrm{~dB}$. A change in perceived location was created by creating an $800 \mu$ s time difference between channels, leading to a sensation of a change in location of approximately $90^{\circ}$. Half of the deviants had an $800 \mu$ s delay in the right channel while the other half was in the left channel. In the gap deviants $7 \mathrm{~ms}$ (including a 1-ms rise and fall) were removed from the middle of the standard stimulus. Stimuli were presented in 3 sequences of 5 minutes each (1845 stimuli) for a total of 15 minutes (5535 stimuli). Each sequence started with a 15 standard tones, followed by a sequence in which every second tine was a standard $(\mathrm{p}=0.5)$ and every other tone was one of the five deviants ( $\mathrm{p}=0.1$ each). One deviant of each category was presented once every five deviants and deviants of the same category were never presented consecutively.

\section{ERP Procedures}

ERPs were recorded with a cap embedded with $\mathrm{Ag}^{+} / \mathrm{Ag}^{+} \mathrm{Cl}^{-}$ electrodes (EasyCap, Herrching-Brieibrunn, Germany) positioned on 32 (see Figure 2) according to the 10-10 system (85). An electrode on the nose served as reference and a ground electrode was positioned at the $\mathrm{AF}_{\mathrm{z}}$ electrode site. Electrodes were placed above and below the right eye to record vertical electrooculographic (VEOG) activity. Electrical recordings were carried out using a Brain Vision QuickAmp ${ }^{\circledR}$ (Brain Products, GmbH, Munich, Germany) amplifier and Brain Vision Recorder $^{\circledR}$ (Brain Products GmbH, Munich, Germany) software. Electrical activity was sampled at $500 \mathrm{~Hz}$, with amplifier bandpass filters set at 0.1 to $100.0 \mathrm{~Hz}$. Electrode impedances were kept below $5 \mathrm{k} \Omega$.

Off-line analysis was performed with Brain Vision Analyzer ${ }^{\circledR}$ software (Brain Products, GmbH, Munich, Germany). For each stimulus, electrical epochs of $500 \mathrm{~ms}$ duration (beginning $100 \mathrm{~ms}$ prior to stimulus onset) were digitally filtered $(0.1-20 \mathrm{~Hz})(86)$, ocular (87) and baseline corrected (relative to the pre-stimulus segment), and only epochs with EEG voltages $\pm 75 \mu \mathrm{V}$ were used for final ERP averages, which were constructed separately for the standard and each deviant stimulus type at each electrode site. Waveforms for the low and high pitch deviants, those for the low- and high-intensity deviants, and those for the right and left location, were averaged together. The mean number of epochs for $\mathrm{MMN}$ averages was not significantly different between deviants, nor were there differences in epoch numbers (for each deviant) across test sessions or between treatment groups.

MMNs elicited by frequency (fMMN), duration (dMMN), intensity (iMMN), gap (gMMN), and location (lMMN) deviants were analyzed with difference waveforms, which were derived by digital point-by-point subtraction of the standard stimulus values from those elicited by each of the deviant stimuli. Grand average waveforms, raw and subtracted, are displayed in Figure 2. MMN amplitude was defined as the most negative peak ( $\pm 5 \mathrm{~ms}$ ) between 120 and $250 \mathrm{~ms}$ at the frontal electrodes $\left(\mathrm{F}_{3}, \mathrm{~F}_{\mathrm{z}}\right.$, $\mathrm{F}_{4}$ ), the sites exhibiting maximum MMN amplitudes. Amplitude of the N1 component (peak negativity between 90 and $120 \mathrm{~ms}$ ) elicited by the standard stimulus was also measured (from $\mathrm{F}_{\mathrm{z}}$ ) as an index of sensory registration, which is typically reduced in chronic SZ (88).

\section{Source Localization}

Intracortical current density $\left(\mathrm{A} / \mathrm{m}^{2}\right)$ measures at peak $\mathrm{MMN}$ activity (based on ERP grand averages) from predefined ROIs was computed using validated (89) exact low-resolution electromagnetic tomography software (eLORETA, version 2081104) (90, 91). eLORETA models the cortical gray matter as a collection of voxels (6239 voxels with a spatial resolution of $\left.5-\mathrm{mm}^{3}\right)$. Relying on the standard electrode positions displayed on the scalp $(92,93)$, the digitized Talairach atlas (94), the average MRI brain template (MMI152) provided by the Montreal Neurological Institute (95) and a cortically restrained solution space, it calculates within a realistic head model (96) the non-unique "inverse" problem by computing a three dimensional distribution of intracortical source activity (with zero location error) at each voxel based on surface-level electrical signals. The original LORETA method has received considerable validation from studies using EEG (97) and more established 
localization methods such as structural and functional MRI (98100) and intracranial electrode recordings (101). Employing the ROI-Extractor tool, the selected ROIs were based on eLORETAdefined Brodmann Areas (BA), and current density data from a single centroid representative voxel of each BA (the voxel closest to the center of the BA mass, which is an excellent representation of the corresponding BA) were extracted for further analysis. This included the pAC (BA 41) and secondary (sAC) auditory cortex (BA 42).

\section{Statistical Analyses}

Statistical analysis was conducted using SPSS version 23 (SPSS Inc., Chicago IL, USA). Two sets of analyses were carried out: 1) the primary set compared data between two groups, including the 13 patients completing the CBTv treatment arm and the 11 patients completing the TAU treatment arm;2) the secondary set combined data from two groups, including the patients assigned to the CBTv treatment arm and the TAU patients who went on to receive $\mathrm{CBTv}$. For the primary analyses, MMNs were assessed with separate mixed analysis of variance (ANOVA) for each deviant, each ANOVA consisting of one between-group factor with 2 levels (CBTv vs. TAU) and two within-group factors, including time (baseline vs. follow-up) and frontal electrode site (left $\left[\mathrm{F}_{3}\right]$, central $\left[\mathrm{F}_{\mathrm{z}}\right]$, and right $\left[\mathrm{F}_{4}\right]$ ). MMN latency (at $\mathrm{F}_{\mathrm{z}}$ only) for each deviant and clinical rating/questionnaire scores were analyzed with similar ANOVAs but with no site factor. Measured as peak negativity in an 80 to $120 \mathrm{~ms}$ window, the N100 amplitude/latency values derived from the standard stimulus were also subjected to similar ANOVAs to determine if CBTv affected simple sensory registration. For the deviants exhibiting significant treatment-induced changes in MMN in the betweengroup analyses, the eLORETA-derived $\mathrm{CD}$ values for the $\mathrm{pAC}$ and sAC were analyzed using ANOVAs involving a betweengroup factor and two within-group factors, including time and ROI (BA41, BA42). For the secondary set of analyses, which assessed measures in the combined CBTv treatment group, ANOVAs did not contain a between-group factor. In order to maintain a constant 5 month period between baseline and follow-up sessions in these analyses, the data from the assessments conducted at the initial follow-up session in the TAU group served as their baseline data. For both sets, regardless of whether significant Greenhouse-Geisser corrected $(\mathrm{p}<0.05)$

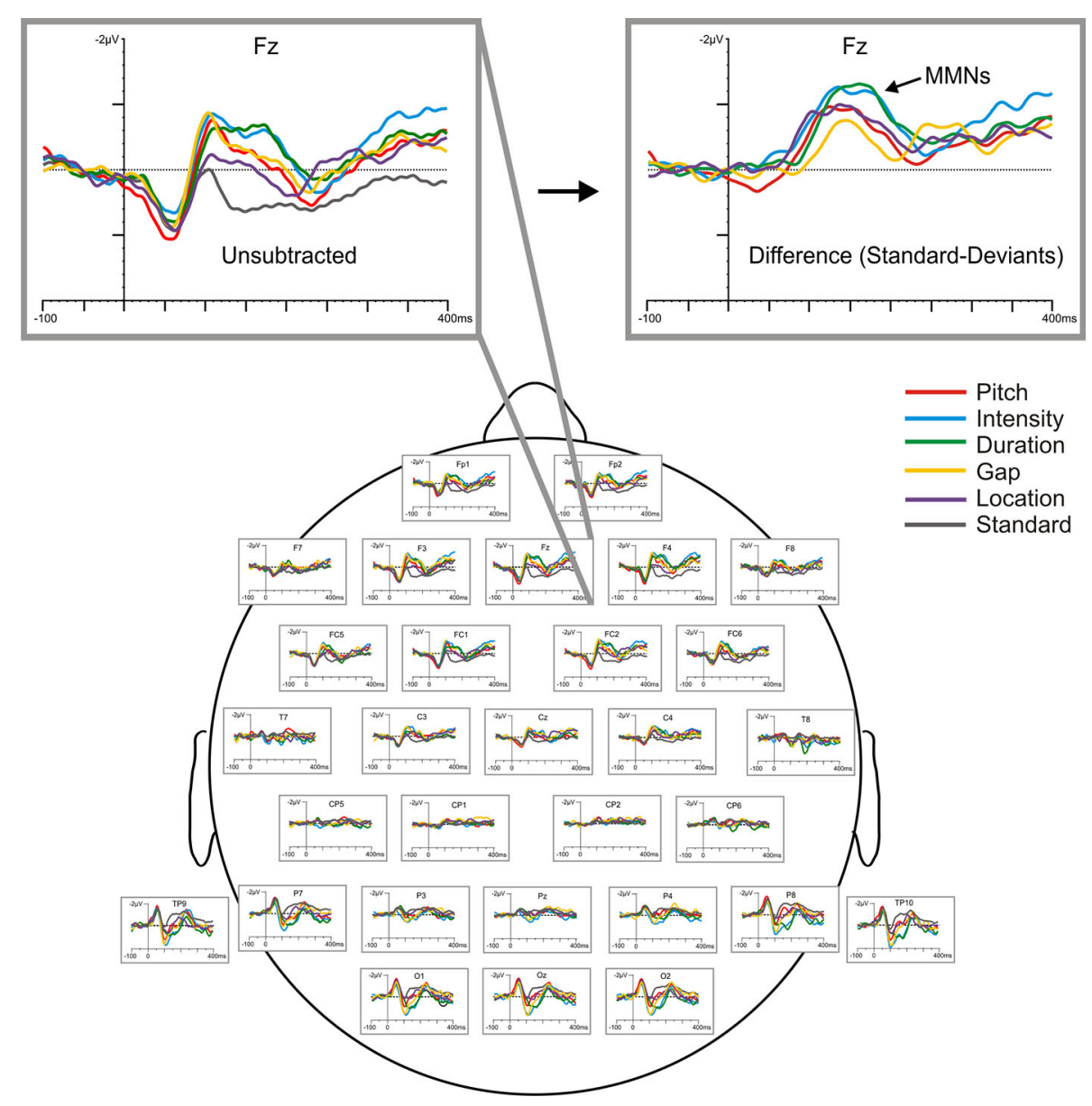

FIGURE 2 | Baseline (raw) grand-averaged ERP waveforms elicited by the standard and five deviant stimuli for all participants ( $N=24)$, shown across scalp sites and highlighted at $\mathrm{F}_{\mathrm{z}}$ with respect to both raw (unsubtracted) and subtracted (deviant minus standard) difference waveforms. 
treatment, time or interaction effects were observed or not, treatment change was assessed via a priori planned comparisons of baseline vs. follow-up data. For the deviant MMNs and CDs exhibiting significant treatment effects in this between-group analysis, Spearman's rho correlation coefficient statistic was used to examine the relationship between changes in electrophysiological measures and changes in clinical/ questionnaire measures, as well as between MMN changes and source localized CD changes. In order to reduce the number of statistical tests, these correlations were assessed only for electrophysiological and clinical/AVH measures showing CBTv treatment effects in the initial set of analyses.

\section{RESULTS}

Of the fourteen patients assigned to CBTv, thirteen (8 males) completed all assessments at baseline and follow-up and provided usable EEG recordings. Of the eleven patients assigned to TAU, ten (6 males) completed all assessments at baseline and follow-up and provided usable EEG recordings. Thus, the attrition rate was $8 \%$, with onset of medical illness (one patient) and unusable EEG data (one patient) accounting for patient-drops. During their subsequent participation in CBTv, only six of the ten patients in the TAU group completed all assessments at baseline (i.e., some data as from their follow-up session post TAU) and at follow-up and provided usable EEG recordings. Patent-drops were due to either change in medication (one patient), or unusable EEG (two patients). The final CBTv and TAU groups were similar in age, gender, year of education, duration of illness, PANSS positive, PANSS negative, PANNS total and PSYRATS total scores (Table 1).

\section{CBTv Effects on Clinical/AVH Symptoms Between-Group Analyses}

The CBTv group did not differ from the TAU group with respect to changes in PANSS positive, negative or general symptoms (Table 1). Similarly, no group differences were observed with respect to changes in the frequency and quality of $\mathrm{AVH}$ symptoms assessed with PSYRATS ratings (Table 1).

For the BAVQ-R, significant time effects were observed for two of the subscale scores, omnipotence $(\mathrm{F}=7.36, \mathrm{df}=1 / 21, \mathrm{p}=$ $0.013)$ and resistance behavior $(\mathrm{F}=4.37, \mathrm{df}=1 / 21, \mathrm{p}=0.049)$. Planned comparisons found these reductions in omnipotence $(\mathrm{p}=0.014)$ and increases in resistance behavior $(\mathrm{p}=0.015)$ ratings between baseline and follow-up to be limited to the CBTv group (Figure 3). Analysis of VAAS rating failed to yield any significant group, time or interaction effects but ratings scores on both the CHOICE severity $(\mathrm{F}=8.08, \mathrm{df}=1 / 21, \mathrm{p}=0.01)$ and CHOICE satisfaction $(\mathrm{F}=12.16, \mathrm{df}=1 / 21, \mathrm{p}=0.002)$ dimensions showed significant time effects, with planned comparisons showing significant changes in severity ( $\mathrm{p}=$ $0.003)$ and satisfaction $(\mathrm{p}=0.008)$ only in the CBTv group (Figure 3).

TABLE 1 | Demographic and clinical measures for treatment groups.

\begin{tabular}{|c|c|c|c|c|}
\hline \multirow[t]{2}{*}{ Demographics } & \multicolumn{2}{|c|}{ CBTv group ( $n=13 ; 7$ males) } & \multicolumn{2}{|c|}{ TAU group ( $n=10 ; 6$ males) } \\
\hline & Mean \pm SD Baseline & Mean \pm SD Follow-up & Mean \pm SD Baseline & Mean \pm SD Follow-up \\
\hline Age (years) & $41.77 \pm 14.69$ & & $47.8 \pm 11.81$ & \\
\hline Education (years) & $4.62 \pm 1.33$ & & $5.5 \pm 1.18$ & \\
\hline Duration of illness (years) & $16.1 \pm 11.64$ & & $21.78 \pm 9.60$ & \\
\hline PSYRATS total & $25.15 \pm 5.38$ & $22.53 \pm 6.21$ & $27.5 \pm 4.62$ & $27.89 \pm 4.76$ \\
\hline \multicolumn{5}{|l|}{ PANSS } \\
\hline Positive Scale & $15.62 \pm 3.31$ & $16.2 \pm 4.41$ & $15.6 \pm 3.53$ & $16.17 \pm 5.94$ \\
\hline Negative Scale & $15.77 \pm 4.78$ & $16.2 \pm 4.59$ & $15.5 \pm 4.17$ & $13.7 \pm 5.28$ \\
\hline General & $33.31 \pm 13.71$ & $30.3 \pm 6.17$ & $31.3 \pm 6.43$ & $31.67 \pm 7.71$ \\
\hline
\end{tabular}

PSYRATS, The Auditory Hallucinations subscale from the Psychotic Symptom Rating Scale; PANSS, Positive and Negative Syndrome Scale.

Follow-up for the PANSS measures included missing data (3 missing cases from the CBTV group and 4 missing cases from the TAU only group).
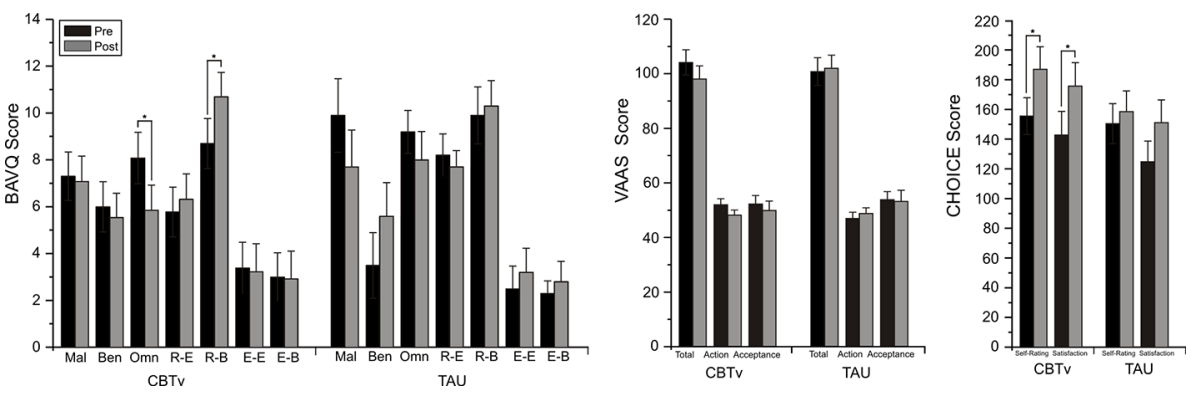

FIGURE 3 | Mean ( \pm SE) rating scores for BAV-Q, VAAS, and CHOICE instruments administered to patients in CBTV and TAU conditions at pre- (baseline) and post-treatment. 


\section{Combined Group Analyses}

Changes from pre- to post-CBTv were not observed for PANSS or VAAS ratings but significant reductions were shown for total PSYRATS ( $p=0.035)$, BAVQ-R omnipotence $(p=0.0013)$ and CHOICE severity $(p=0.009)$ rating scores (Figure 4).

\section{CBTv Effects on MMN/N100 Amplitude/ Latency \\ Between-Group Analyses}

Analysis of frontal amplitudes did not reveal any significant group, treatment, or time effects for MMN elicited by intensity, duration, gap or location deviants. A significant treatment $\mathrm{x}$ time interaction $(\mathrm{F}=15.78, \mathrm{df}=1 / 40, \mathrm{p}=0.001)$ was shown for the pitch deviant, with planned comparisons revealing significant increases in pMMN amplitudes in the CBTv group at follow-up compared to baseline $(\mathrm{p}=0.001)$ as well as greater $\mathrm{pMMN}$ amplitudes in CBTv group compared to TAU group $\mathrm{p}=0.043$ ) at follow-up (Figure 5). Analysis of MMN latency yielded a significant treatment $\mathrm{x}$ time interaction for the gap deviant $(\mathrm{F}=4.41, \mathrm{df}=1 / 25, \mathrm{p}=0.049)$ with planned comparisons showing a reduced (earlier) gMMN latency (Figure 6) in the CBTv group ( $\mathrm{p}=0.019)$ at follow-up $(\mathrm{M}=148.68 \mathrm{~ms}, \mathrm{SE} \pm 6.86)$ compared to baseline $(\mathrm{M}=164.02 \mathrm{~ms}, \mathrm{SE} \pm 6.92)$. Neither the amplitude nor latency of N100 were affected by treatment.

\section{Combined Group Analyses}

A significant time effect was observed only for the pitch deviant $(\mathrm{F}=14.68, \mathrm{df}=1 / 18, \mathrm{p}=0.001)$, with $\mathrm{pMMN}$ amplitudes showing an increase at follow-up compared to baseline (Figure 7). Analyses of the duration deviant yielded a significant time $\mathrm{x}$ electrode interaction $(\mathrm{F}=9.12$, $\mathrm{df}=1 / 36$, $\mathrm{p}=0.002$ ), with comparisons of left frontal (F3) amplitude showing a greater $\mathrm{dMMN}$ amplitude $(\mathrm{p}=0.029)$ at follow-up compared to baseline (Figure 7). For MMN latency, analysis showed a significant time effect for the duration deviant $(\mathrm{F}=6.71, \mathrm{df}=1 / 18, \mathrm{p}=0.018)$, with $\mathrm{dMMN}$ exhibiting a shorter latency $(\mathrm{M}=153.01 \mathrm{~ms}, \mathrm{SE} \pm 7.11)$ at follow-up compared to baseline $(\mathrm{M}=166.46 \mathrm{~ms}, \mathrm{SE} \pm 6.03)$ latency
(Figure 7). No treatment effects were observed for N100 amplitude or latency.

\section{CBTv Effects on Source Localized CD \\ Between-Group Analyses}

For the pMMN, analysis of localized CD yielded a significant region effect $(F=33.53, \mathrm{df}=1 / 21, \mathrm{p}=0.001)$, with $\mathrm{CD}$ of the $\mathrm{sAC}$ being greater than $\mathrm{CD}$ of the $\mathrm{pAC}$. A significant treatment $\mathrm{x}$ time $\mathrm{x}$ hemisphere interaction was also evidenced $(\mathrm{F}=11.62, \mathrm{df}=1$ / $21, \mathrm{p}=0.008)$, with planned comparisons finding increases $(\mathrm{p}=0.008)$ in CD in the left hemisphere of the CBTv group at follow-up compared to baseline (Figure 8).

\section{Combined Group Analyses}

Analysis of $\mathrm{CD}$ associated with the pMMN showed significant $(\mathrm{F}=17.85, \mathrm{df}=1 / 18, \mathrm{p}=0.001)$ region effect, with $\mathrm{CD}$ in the $\mathrm{sAC}$ being greater than $\mathrm{CD}$ in the $\mathrm{pAC}$. In significant time $(\mathrm{F}=5.38$, $\mathrm{df}=1 / 18, \mathrm{p}=0.032)$ and time $\mathrm{x}$ hemisphere interaction effects $(\mathrm{F}=8.27, \mathrm{df}=1 / 18, \mathrm{p}=0.010)$, planned comparisons showed significant overall increases $(\mathrm{p}=0.002)$ in $\mathrm{CD}$ of the left auditory cortex in the CBTv group at follow-up compared to baseline (Figure 9).

\section{Relationships Between Symptoms and MMN/CD}

In the initial CBTv group, changes in the pMMN amplitude (from baseline to follow-up) were positively correlated with changes in the resistance emotion subscale of the BAVQ-R $(\mathrm{r}=0.64, \mathrm{p}=0.029)$, and negatively correlated with the total $(\mathrm{r}=-0.76, \mathrm{p}=0.002)$ and both the activation $(\mathrm{r}=-0.82$, $\mathrm{p}=0.001)$ and acceptance $(\mathrm{r}=-0.70, \mathrm{p}=0.008)$ scores of the VAAS (Figure 10). Treatment-induced changes in symptoms were found to be related to treatment-induced changes in $\mathrm{CD}$ of the auditory cortex but only in the left pAC. In this region of the auditory cortex, $\mathrm{CD}$ changes were positively correlated with changes in the benevolence $(r=0.63, p=0.021)$, resistance emotion $(\mathrm{r}=0.55, \mathrm{p}=0.05)$ and engagement behavior subscale scores $(r=0.54, p=0.04)$ of the BAVQ-R, and negatively
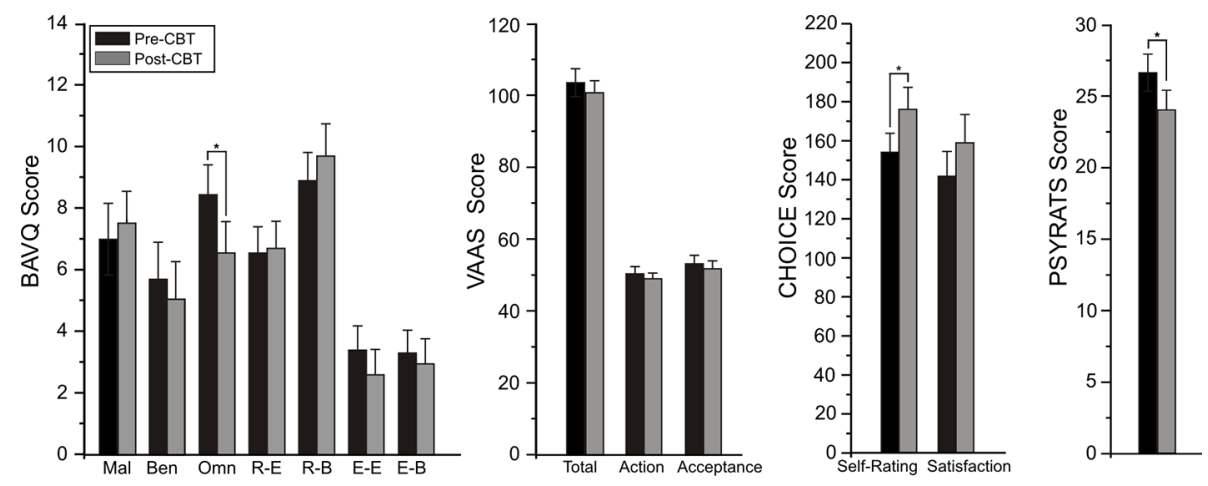

FIGURE 4 | Mean ( \pm SE) pre- (baseline) and post-treatment rating scores for BAV-Q, VAAS, and CHOICE instruments administered to all patients (N=22) completing CBTv. 


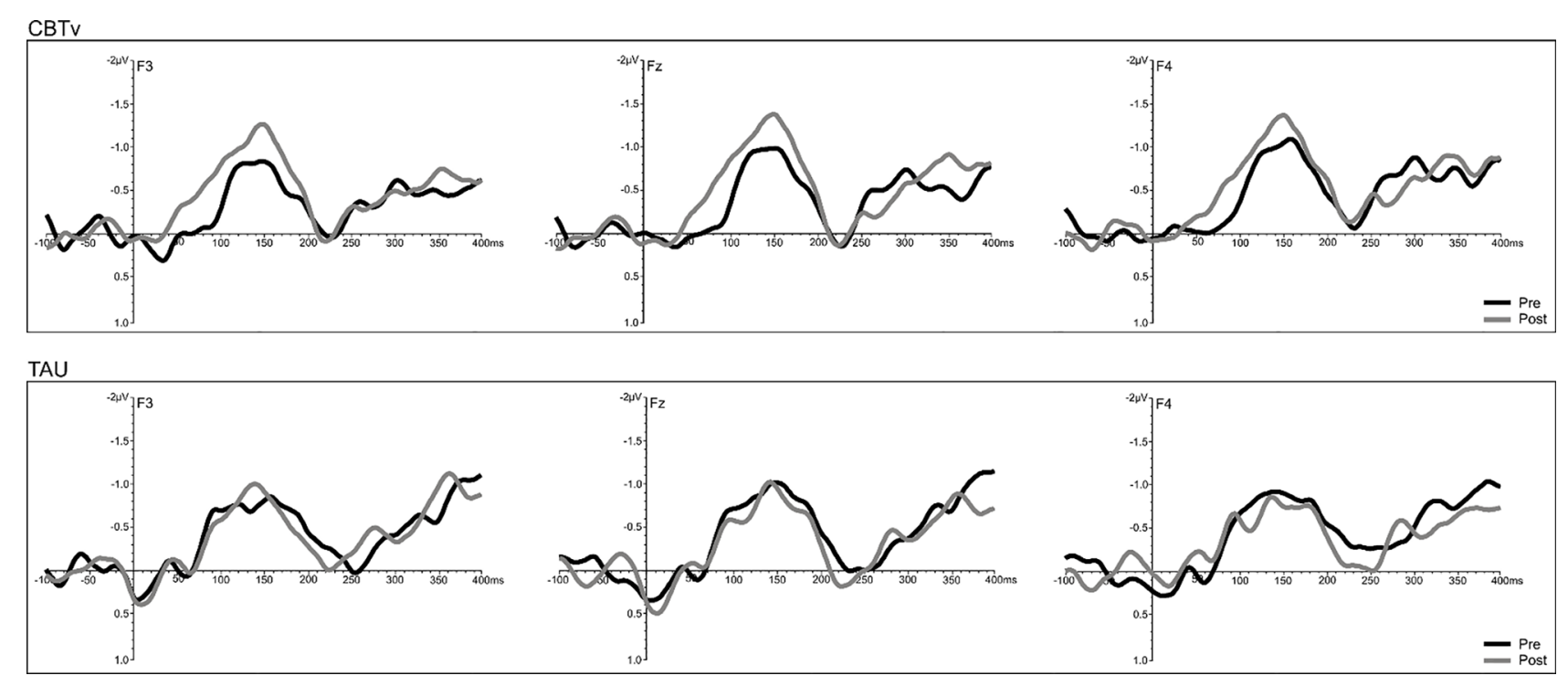

FIGURE 5 | Grand-averaged subtracted pitch deviant waveforms at frontal $\left(F_{3}, F z, F_{4}\right)$ sites recorded pre- (baseline) and post-treatment in patients assigned to CBTV and TAU conditions.

correlated with changes of the total VASS $(r=-0.68, \mathrm{p}=0.01)$ score (Figure 11).

\section{Relationships Between MMN and CD}

In the initial CBTv group, correlations between the treatment changes in the pMMN and the associated $\mathrm{CD}$ were limited to the left hemisphere, with increases in $\mathrm{AMMN}$ at F3 being positively correlated with increases in $\mathrm{CD}$ in the left $\mathrm{pAC}(\mathrm{r}=0.58, \mathrm{p}=$ $0.01)$, left $\mathrm{sAC}(\mathrm{r}=0.53, \mathrm{p}=0.02)$ and in combined left auditory cortical regions $(\mathrm{r}=0.55, \mathrm{p}=0.01)$.

\section{DISCUSSION}

This pilot study shows changes in EAIP during a pure tone auditory oddball paradigm in a sample of patients with $\mathrm{SZ}$ and persistent AVHs, attributable to the effects of CBTv. Compared to patients receiving TAU in the initial analyses, the patients who underwent CBTv showed significantly greater increases in auditory deviance detection as evidenced in enhanced MMN response to pitch deviants and faster (earlier latency) MMN responses to auditory gap deviants at treatment follow-up. These changes occurred independently of general psychiatric symptoms (PANSS) and changes in sensory registration (N100). The patients that received therapy also showed significant increases in activation (CD) of the left auditory cortex during the processing of auditory pitch deviants. Although CBTv did not affect self-reports of the frequency or quality of AVHs (PYSRATS), or the severity of psychotic symptoms (PANSS), it improved patients' perceptions and behavioral response to AVHs (BAVQ-R), and CBTv was thought by patients to be associated with better outcome (CHOICE) than TAU. Even more interestingly, these clinical changes together with self-reported improvements in patient
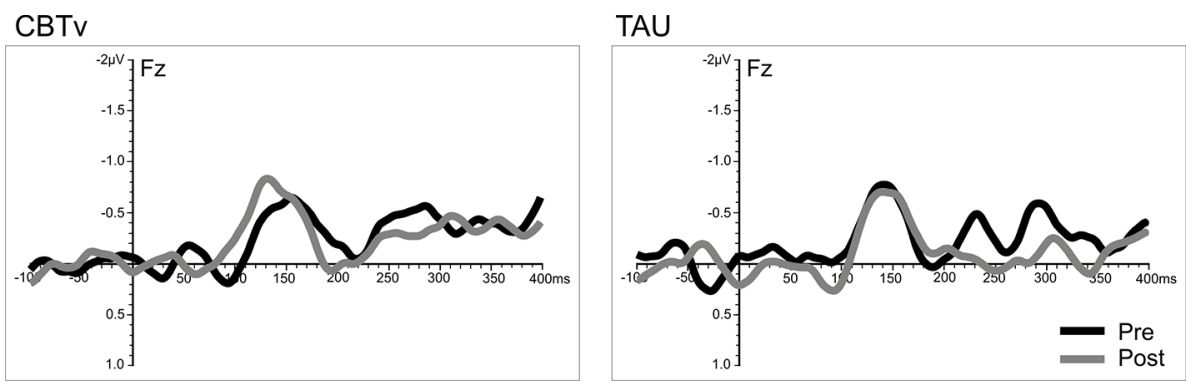

FIGURE 6 | Grand-averaged subtracted gap deviant waveforms at mid-frontal site (Fz) recorded pre- (baseline) and post-treatment in patients assigned to CBTV and TAU conditions. 


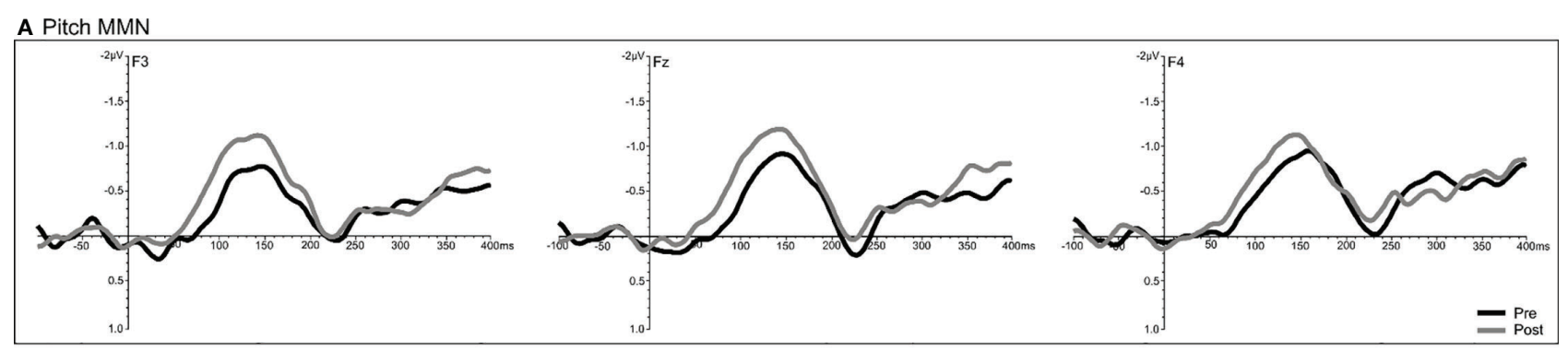

\section{B Duration MMN}

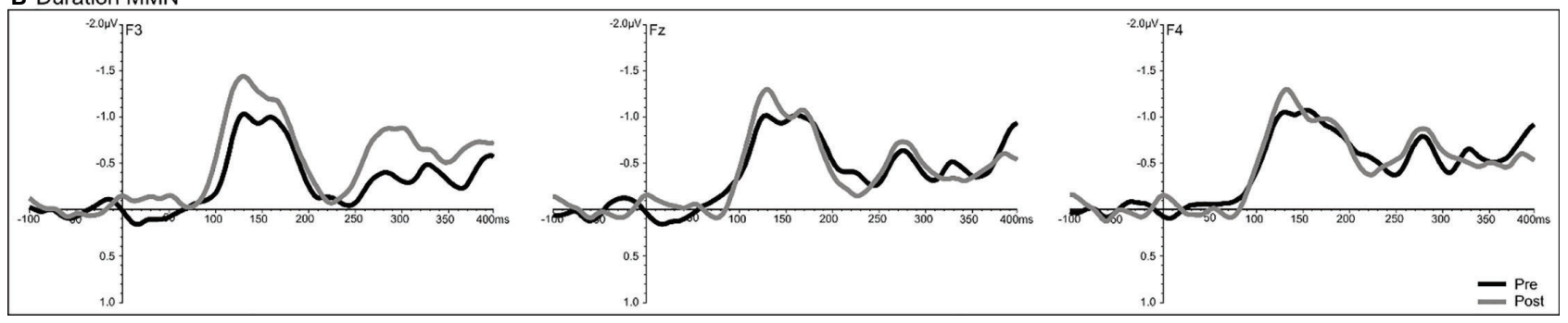

FIGURE 7 | Grand-averaged subtracted pitch (A) and duration (B) deviant waveforms at frontal $\left(F_{3}, F_{2}, F_{4}\right)$ sites recorded pre- (baseline) and post-treatment in all patients ( $N=22)$ completing CBTv.

beliefs about AVHs (VAAS) at CBTv follow-up were associated with increases in MMN response and left auditory cortex activation to pitch deviants.

Targeting the cognitive appraisals, perceptions, and beliefs concerning the nature of psychotic symptoms, both individual CBT (41) and group CBT in SZ (102) have been effective in alleviating positive psychotic symptoms as a whole (i.e.

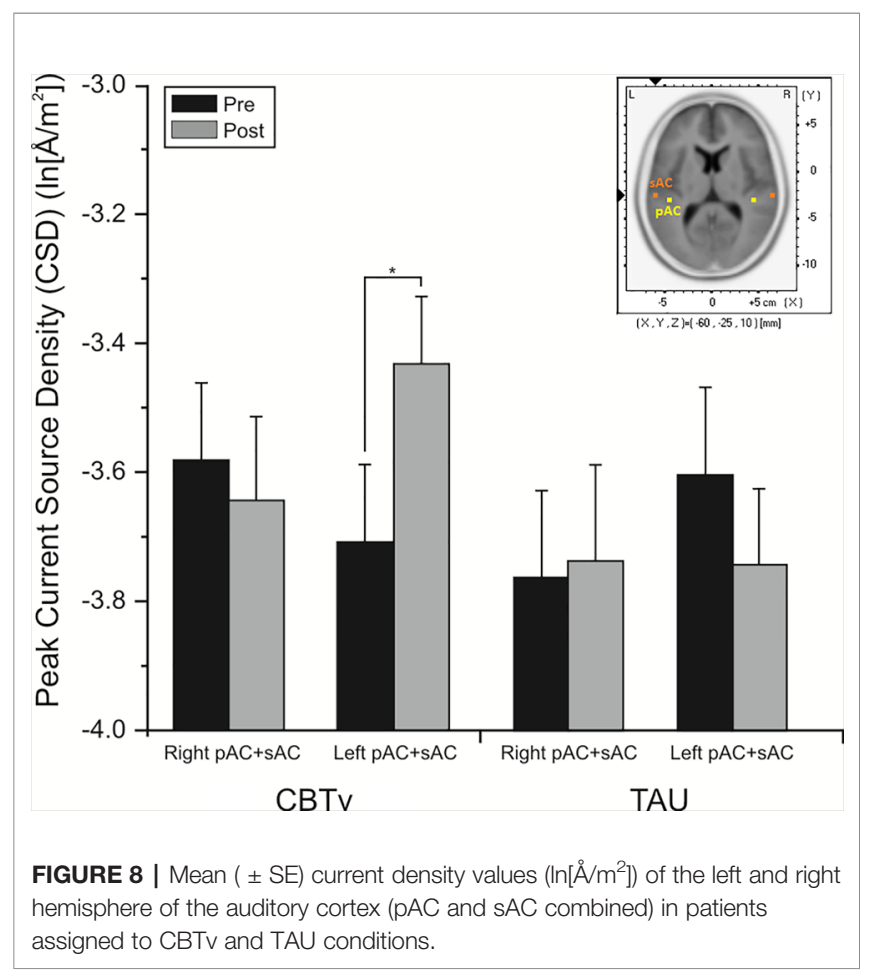

hallucinations and delusions) but, as with our own negative findings, the efficacy of CBT interventions specific for hallucinations has been mixed in regards to reductions in the frequency and severity of these symptoms (103, 104). Similar mixed findings in SZ have been reported in CBT trials with added ACC and ATT techniques (105). Our augmented CBTv

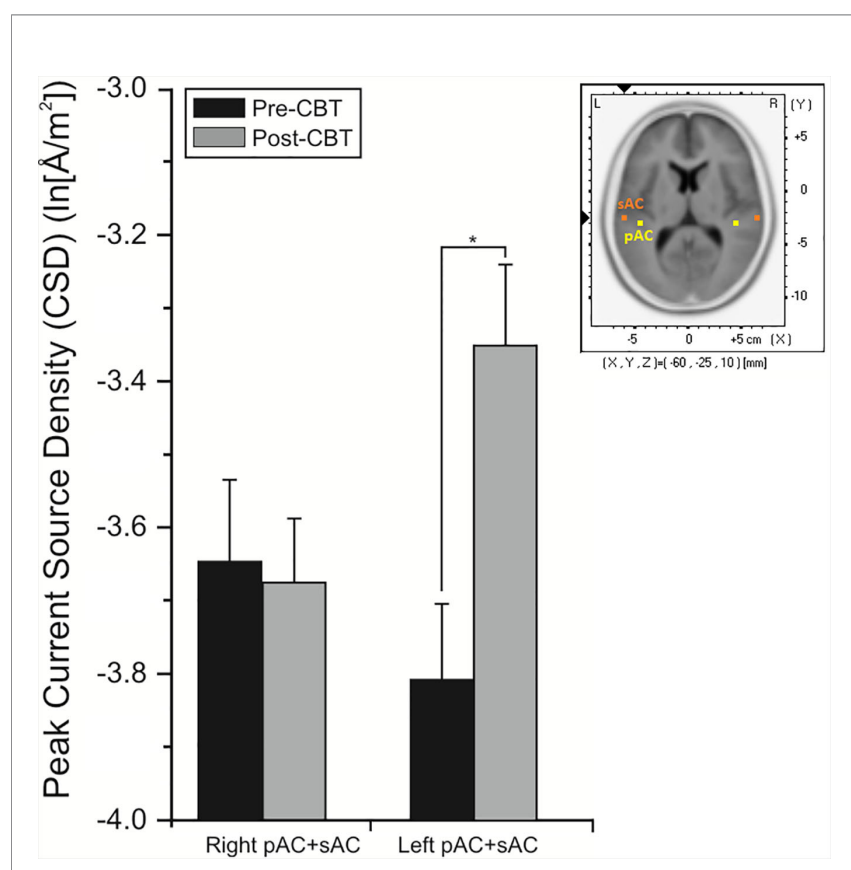

FIGURE 9 | Mean ( \pm SE) current density values $\left(\ln \left[\AA ̊ \Omega / m^{2}\right]\right)$ of the left and right hemisphere of the primary and secondary auditory cortex in all patients ( $\mathrm{N}=22)$ completing CBTV. 

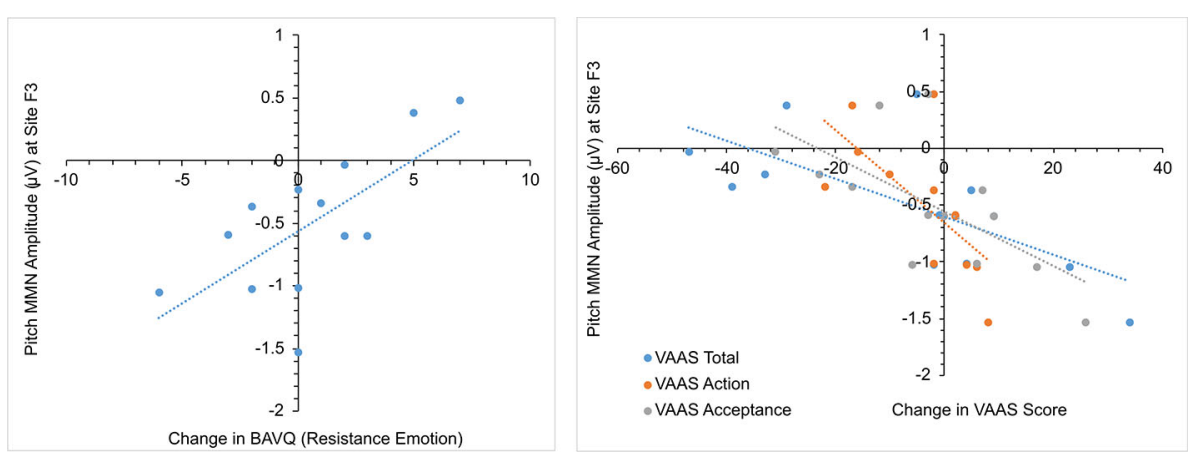

FIGURE 10 | Scatterplots showing relationships between pitch MMN amplitude change and BAV-Q and VAAS ratings change in the initial group receiving CBTV.
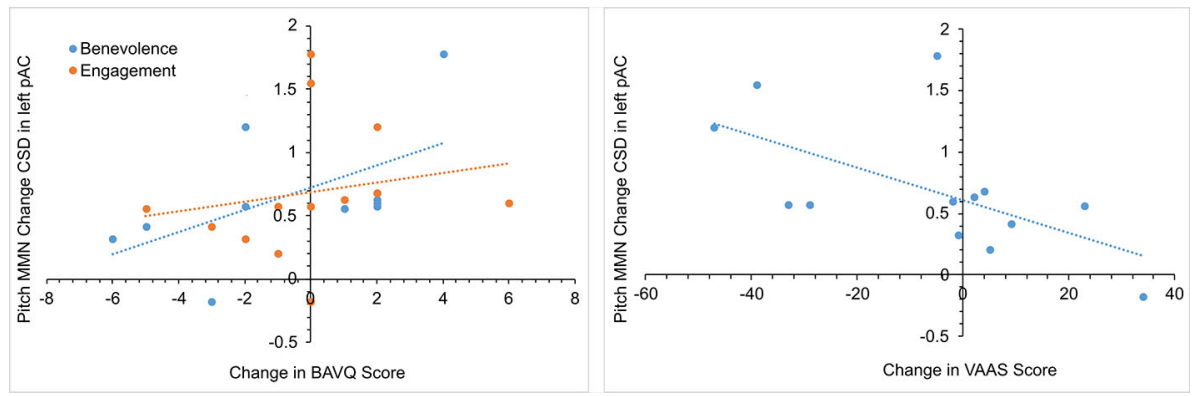

FIGURE 11 | Scatterplots showing the relationships between changes in pitch MMN current density (CSD) in the left primary auditory cortex (pAC) and BAVQ-R and VAAS rating changes in the initial group receiving CBTV.

did however affect AVH-related beliefs and behavioral reactions to hallucinations, shown by reductions in BAVQ-R Omnipotence and BAVQ-R Resistance Behavior scores, which may in part have accounted for patients' favorable outcome perception (CHOICE) with this therapy.

Reductions in conviction of beliefs about the power/ authority (omnipotence) of voices and compliance to voices have been observed in previous group CBTp trials (105-107) and are clinically important considering that our patients' hallucinations have failed to respond to effective antipsychotic drugs. Employing specialized interventions like CBTv to target beliefs about voice omnipotence, particularly with respect to commanding aspects of the voices, which have been linked to a range of dangerous behaviors (aggression, violence, self-harm, and suicide), is a therapeutically relevant goal as voice omnipotence predicts compliance to hallucinations, and reductions in these beliefs about voices are associated with reduced cognitive functioning given their negative relationship with exogenous attentional processes $(26,108-110)$.

Consistent with our hypotheses that dampening the impact of internal voice processing with CBTv would result in the enhanced sensory processing of external auditory stimuli, improvements in EAIP with therapy were evidenced by larger pMMN and shorter gMMN responses in SZ patients with persistent AVHs. Rarely examined with other deviant types, abnormal deviance detection in SZ has been most frequently documented with pitch and duration, and occasionally with intensity changes in simple sound stimuli $(17,111,112)$. While pMMN is thought to be sensitive to illness duration/disease progression, the $\mathrm{dMMN}$ behaves more as a trait index and as a valid endophenotype reflecting greater vulnerability to illness (17). Although not consistently observed, attenuated pMMN, $\mathrm{dMMN}$, and $\mathrm{iMMN}$ have been observed in our laboratory in hallucinating (vs. non-hallucinating) SZ patients and have been correlated with hallucinatory severity ratings $(19-22,113,114)$.

Possibly reflecting contributions from bilateral prefrontal cortices, the auditory MMN response to both simple sound and speech deviants is mainly dependent on synaptic plasticity mediated by glutamatergic $N$-methyl-D-aspartate (NMDA) receptors in the auditory cortex (115-117). Although also shown with the detection of duration deviants in our combined group analysis, the more reliable improvements in deviance detection with CBTv were observed in response to the pitch deviant, with pMMN being shown to be increased in both sets of analyses along with pitch CD in auditory cortices. Not always associated with auditory hallucinatory experiences, SZ is associated with deficits in the perception of a broad range of auditory features, including pitch discrimination as measured in 
tone-matching tasks (118). Dependent on the low-level acoustic features or type of complex naturalistic sound, the neural representation and processing of acoustic stimuli is confined to different regions within the human auditory system. The neural mechanisms underlying pitch perception are still largely debated but are assumed to involve a hierarchy of pitch processing steps, starting in the subcortical structures and terminating at the cortical level, where perceived pitch (variations) is most likely encoded (119). Certain areas of the auditory cortex are specifically sensitive to pitch, and although the locations are still another debate (120), previous functional neuroimaging has identified pitch-coding regions, including anterior-lateral pAC (on Heschl's gyrus [HG]) and adjacent sAC processing areas (on the superior temporal gyrus [STG]) (121), and extending to the planum temporale during the passive influences of infrequent pitch changes $(122,123)$. Increased pMMN (and dMMN) amplitudes with CBTv in patients experiencing persistent AVHs is consistent with brain volumetric studies showing negative relationships between left hemisphere HG/STG volume and both hallucination severity $(122,123)$ and MMN amplitude in SZ patients (124).

Changes in the appraisal of and response to AVHs with CBTv were associated with enhanced pMMN amplitudes and selective increases in pitch CD in the left pAC, and are consistent with the current status of AVHs in that they implicate speech perception areas in the left temporal lobe, improving perception of and attention to external sounds. These relationships between changes in auditory neural responsiveness and AVH symptom ratings are in line not only with structural studies showing the left pAC gray matter volume reduction in SZ to be associated with AVH severity $(122,123,125)$, but also with functional neuroimaging studies confirming a "paradoxical" brain activation in relation to AVHs - the left pAC evidencing increased activation (during silent rest) in the absence of an external stimulus, and decreased activation in the presence of an external auditory stimulus (11). Although the specific brain mechanisms underlying these alterations are not understood, it is reasonable to speculate that they may be mediated by changes in glutamate neurotransmission. Aberrant glutamate levels in temporal and prefrontal cortical areas are found in SZ with frequent and severe hallucinations $(126,127)$ and glutamate receptor mediated synaptic plasticity in the pAC, as indexed by MMN alteration, is compromised in patients with SZ and particularly in patients with AVHs. Although not necessarily affecting tonic glutamate levels, changes in MMN responsiveness to pitch deviance with CBTv and adjunctive ATT and ACC techniques may indicate an increased ability to adequately modify synaptic plasticity in response to auditory (glutamate) neurotransmission resulting from external auditory stimulation.

The co-occurrence of altered ratings of beliefs/responses to AVHs together with changes in neural response (pMMN) to auditory deviance, which was shown to be statistically correlated with changes in beliefs, behavioral/emotional (BAVQ-R) and coping response (VAAS) to hallucinations, may be tentatively explained by the influence of CBTv on a common underlying NMDA receptor-mediated process-prediction error signaling.
In a predictive coding framework, predictive coding is viewed as a hierarchical information processing model which posits interactions between lower-order (bottom-up) perceptual signals and higher-order cognitive processes in a dynamic, interactive fashion to generate predictions about the environment and compare incoming stimuli with these predictions $(128,129)$. Within this model, neural responses to stimuli that match predictions are suppressed, while stimuli that are unexpected, violating these predictions, trigger a mismatch "prediction error" signal, which signals that updating of expectations is required to accommodate the discrepant stimuli (130-132). It has been proposed that in hallucinating patients, excess aberrant spontaneous activation of the auditory sensory cortex may be confused by the brain with activity typically seen with external auditory stimulation, leading to erroneous expectations of a perceptual event (predictive coding failure) with the brain inferring externally located voices which in turn leads to a false (AVH) perception $(129,133,134)$. Predictive tone signaling has been observed at the earliest levels of auditory cortical hierarchy-in the pAC (135). The MMN is hypothesized to reflect a prediction error signal (e.g. the properties of the deviant stimulus do not match the predictive model formed by the train of preceding standards, thus the model must be updated in order to improve predictive accuracy) (136), which is attenuated during NMDA receptor antagonist treatment with ketamine (137-139) and can be used to examine abnormalities in predictive coding. In a roving standard MMN paradigm, which allows for optimal evaluation of prediction errors (140), MMN deficits in SZ have reflected attenuated prediction error signaling (141). This is particularly pronounced in hallucinating (v. nonhallucinating) patients and consistent with a predictive coding account of hallucinations in SZ (142).

Hallucinatory experiences are associated with hyperactivation of the primary and secondary sensory areas, possibly due to dysregulation related to frontal lobe hypo-activation. Different brain mechanisms appear to underlie the clinical effects of pharmacotherapy and psychotherapy $(143,144)$. It has been argued that psychotherapeutic approaches such as CBT may exert their affects by gaining control of the function of particular circuits, such as changes in appraisal, control of attention, modulation of interceptive processes, and may involve key nodes, such as anterior cingulate and medial prefrontal areas (involved in error detection and conflict monitoring), dorsolateral prefrontal cortex areas (involved in cognitive control/working memory), and insula (interceptive sensitivity) (105). We can also speculate that different psychotherapeutical strategies may have different brain effects within a circuit. In our augmented treatment protocol, CBT and AAC focused on controlling the emotional response to hallucinations might be based on decreasing endogenous brain activity in the temporal/limbic areas, while ATT focused on auditory perceptual processes might be based on increasing externally induced brain activity in these same regions. Although not directly comparable with ATT, targeted cognitive training (TCT) of the auditory system in SZ patients has been shown to drive plasticity in cortical activation patterns related to 
both sensory representations as well as higher order cognitive processes $(145,146)$. In patients with SZ, TCT has not only produced significant improvements in auditory perception and learning, which was predicted by MMN (147), but also increases in verbal learning and reductions in AVHs $(148,149)$. Improvements in higher order auditory processing gained through TCT in SZ are dependent on the severity of basic auditory deficits (150). Given that the MMN response to deviant sounds has also been shown to have a direct mediating effect on cognition and functional outcome in SZ patients (151), future research may want to examine the effects of pairing CBTv with TCT aimed at improving auditory discrimination as a potential optimal strategy that would benefit both $\mathrm{AVH}$ symptoms and cognitive and psychosocial functioning.

\section{LIMITATIONS}

This study has several limitations. Regarding adherence to CBTv, a fidelity/treatment response scale was not used, and future research would benefit from audiotaping (with consent) and using a fidelity measure and independently trained raters. Although the results are relatively consistent across the two sets of analyses, they require replication in a larger sample. Patients were randomized to CBTv and TAU vs TAU only conditions but blindness was not a component of the study design and should be an aim in future work. Concurrent antipsychotic treatment may have influenced the results, but both groups of patients were receiving similar treatments. In order to reduce Type 1 error rates our source analysis with eLORETA was limited to two ROI, and additional studies are required to examine $\mathrm{CBTv}$ effects on non-auditory brain regions, especially frontal areas which are thought to contribute to MMN generation during deviance detection and to interact with auditory cortices in producing AVHs. External auditory stimulation for MMN generation was limited to pure tone deviants and additional studies need to examine CBTv effects on the processing of complex natural sounds including MMN response to speech deviants, which appear to be particularly sensitive to EAIP dysfunction in patients with SZ $(21,152)$ and as with MMN to response to pure tone deviants are reflective of NMDA receptor-mediated neurotransmission in auditory cortices (117). Functional neuroimaging has shown that the neural encoding of natural sounds (e.g., speech, voice) entails the formation of multiple representations of sound spectrograms with different degrees of spectral and temporal resolution (152154). Combining the superior temporal and spatial resolution of EEG and fMRI techniques, respectively, to image neural activity during resting-state (absence of external auditory stimulation) and in response to behaviorally relevant, real-world sound stimuli would be an optimal strategy for achieving a more complete picture of brain mechanisms involved in $\mathrm{AVH}$ responsiveness to CBTv in SZ patients (155-160).

Finally, we assessed responsivity to external auditory stimulation on the neural level and there is a need to incorporate behavioral assessments (e.g., tone-matching and dichotic listening tasks) in order to examine performance changes associated with auditory processing. Optimally, these would be complemented with tests assessing changes in cognitive and functional outcome with CBTv.

\section{CONCLUSION}

In conclusion, we have shown for the first time significant changes in MMN responsiveness to external auditory deviants in SZ patients undergoing cognitive therapy for persistent auditory hallucinations. Correlated with improvements in patient's response to hallucinations, these neural findings improve our understanding of how psychotherapy may benefit patients with $\mathrm{AVH}$, possibly by shifts in perceptual processing from internal distressing auditory (voices) stimulation to potentially relevant changes in external auditory stimuli. As these present observations were captured in a relatively small test sample, confirmation of these observations in larger studies integrating biomarkers like MMN to elucidate treatment effect mechanisms will help to increase our understanding of and ability to personalize psychotherapeutic approaches such as CBTv $(153,154)$.

\section{DATA AVAILABILITY STATEMENT}

The datasets generated for this study are available on request to the corresponding author.

\section{ETHICS STATEMENT}

The studies involving human participants were reviewed and approved by Dominique Bourget, Acting Chair, University of Ottawa Institute of Mental Health Research REB. The patients/ participants provided their written informed consent to participate in this study.

\section{AUTHOR CONTRIBUTIONS}

VK and NW were responsible for the conceptualization of the study, NW lead the CBT for patients and was assisted by DS. Recruitment was the responsibility of $\mathrm{AL}$ and coordination, testing, and data analysis were performed by HB, DS, SS, and $\mathrm{AB}$. Manuscript was prepared by VK and figures and tables were prepared by SS.

\section{FUNDING}

This work was supported by a grant from the University of Ottawa Medical Research Fund to NW, VK, and AL. 


\section{REFERENCES}

1. Andreasen NC, Flaum M. Schizophrenia: the characteristic symptoms. Shizophr Bull (1991) 17(1):27-49. doi: 10.1093/schbul/17.1.27

2. Jones S. Do We Need Multiple Models of Auditory Verbal Hallucinations? Examining the Phenomenological Fit of Cognitive and Neurological Models. Schizophr Bull (2010) 36(3):566-75. doi: 10.1093/schbul/sbn129

3. Tracy D, Shergill D. Imaging auditory hallucinations in schizophrenia. Acta Neuropsychiat (2006) 18(2):71-8. doi: 10.1111/j.1601-5215.2006.00129.x

4. Allen P, Laroi F, McGuire PK, Aleman A. The hallucinating brain: a review of structural and functional neuroimaging studies of hallucinations. Neurosci Biobehav Rev (2008) 32(1):175-91. doi: 10.1016/j.neubiorev.2007.07.012

5. Kuhn S, Gallinat J. Quantitative meta-analysis on state and trait aspects of auditory verbal hallucinations in schizophrenia. Schizophr Bull (2012) 38 (4):779-86. doi: 10.1093/schbul/sbq152

6. Allen P, Modinos G, Hubl D, Shields G, Cachia A, Jardri R, et al. Neuroimaging auditory hallucinations in schizophrenia: from neuroanatomy to neurochemistry and beyond. Schizophr Bull (2012) 38 (4):695-703. doi: $10.1093 / \mathrm{schbul} / \mathrm{sbs} 066$

7. Boly M, Balteau C, Schnakers C, Degueldre C, Moonen G, Luxen A, et al. Baseline brain activity fluctuations predict somatosensory perception in humans. PNAS (2007) 104(29):12187-92. doi: 10.1073/pnas.0611404104

8. Hesselmann G, Kell CA, Kleinschmidt A. Ongoing activity fluctuations in hMT+ bias the perception of coherent visual motion. J Neurosci (2008) 28 (53):14481-5. doi: 10.1523/JNEUROSCI.4398-08.2008

9. Northoff G, Qin P, Nakao T. Rest-stimulus interaction in the brain: a review. Trends Neurosci (2010) 33(6):277-84. doi: 10.1016/j.tins.2010.02.006

10. Sadaghiani S, Hesselmann G, Kleinschmidt A. Distributed and antagonistic contributions of ongoing activity fluctuations to auditory stimulus detection. J Neurosci (2009) 29(42):13410-7. doi: 10.1523/JNEUROSCI.2592-09.2009

11. Kompus K, Westerhausen R, Hugdahl K. The "paradoxical" engagement of the primary auditory cortex in patients with auditory verbal hallucinations: a meta-analysis of functional neuroimaging studies. Neuropsychologia (2011) 49(12):3361-9. doi: 10.1016/j.neuropsychologia.2011.08.010

12. Woodruff PW, Wright IC, Bullmore ET, Brammer M, Howard RJ, Williams SC, et al. Auditory hallucinations and the temporal cortical response to speech in schizophrenia: a functional magnetic resonance imaging study. Am J Psychiatry (1997) 154(12):1676-82. doi: 10.1176/ajp.154.12.1676

13. Ford JM, Roach BJ, Jorgensen KW, Turner JA, Brown GG, Notestine R, et al. Tuning in to the voices: a multisite FMRI study of auditory hallucinations. Schizophr Bull (2009) 35(1):58-66. doi: 10.1093/schbul/sbn140

14. Rissling AJ, Light GA. Neurophysiological measures of sensory registration, stimulus discrimination, and selection in schizophrenia patients. Curr Top Behav Neurosci (2010) 4:283-309. doi: 10.1007/7854_2010_59

15. Turetsky BI, Calkins ME, Light GA, Olincy A, Radant AD, Swerdlow NR. Neurophysiological endophenotypes of schizophrenia: the viability of selected candidate measures. Schizophr Bull (2007) 33(1):69-94. doi: $10.1093 / \mathrm{schbul} / \mathrm{sbl} 1060$

16. Patterson JV, Hetrick WP, Boutros NN, Jin Y, Sandman C, Stern H, et al. P50 sensory gating ratios in schizophrenics and controls: a review and data analysis. Psychiatry Res (2008) 158(2):226-47. doi: 10.1016/j.psychres.2007.02.009

17. Naatanen R, Kahkonen S. Central auditory dysfunction in schizophrenia as revealed by the mismatch negativity (MMN) and its magnetic equivalent MMNm: a review. Int J Neuropsychopharmacol (2009) 12(1):125-35. doi: $10.1017 /$ S1461145708009322

18. Smith DM, Grant B, Fisher DJ, Borracci G, Labelle A, Knott VJ. Auditory verbal hallucinations in schizophrenia correlate with P50 gating. Clin Neuriphysiol. (2013) 124(7):1329-35. doi: 10.1016/j.clinph.2013.02.004

19. Fisher DJ, Labelle A, Knott VJ. The right profile: mismatch negativity in schizophrenia with and without auditory hallucinations as measured by a multi-feature paradigm. Clin Neurophysiol (2008) 119(4):909-21. doi: 10.1016/j.clinph.2007.12.005

20. Fisher DJ, Grant B, Smith DM, Borracci G, Labelle A, Knott VJ. Effects of auditory hallucinations on the mismatch negativity (MMN) in schizophrenia as measured by a modified 'optimal' multi-feature paradigm. Int J Psychophysiol (2011) 81(3):245-51. doi: 10.1016/ j.ijpsycho.2011.06.018
21. Fisher DJ, Labelle A, Knott VJ. Auditory hallucinations and the mismatch negativity: processing speech and non-speech sounds in schizophrenia. Int $J$ Psychophysiol. (2008) 70(1):3-15. doi: 10.1016/j.ijpsycho.2008.04.001

22. Fisher DJ, Labelle A, Knott VJ. Auditory hallucinations and the P3a: attention-switching to speech in schizophrenia. Biol Psychol (2010) 85 (3):417-23. doi: 10.1016/j.biopsycho.2010.09.003

23. Hubl D, Koenig T, Strik WK, Garcia LM, Dierks T. Competition for neuronal resources: how hallucinations make themselves heard. $\mathrm{Br} J$ Psychiatry (2007) 190:57-62. doi: 10.1192/bjp.bp.106.022954

24. Javitt D, Sweet R. Auditory dysfunction in schizophrenia: integrating clinical and basic features. Nat Rev Neurosci (2015) 16(9):535-50. doi: 10.1038/ nrn 4002

25. Birchwood M, Gilbert P, Gilbert J, Trower P, Meaden A, Hay J, et al. Interpersonal and role-related schema influence the relationship with the dominant 'voice' in schizophrenia: a comparison of three models. Psychol Med (2004) 34(8):1571-80. doi: 10.1017/S0033291704002636

26. Chadwick P, Birchwood M. The omnipotence of voices. A cognitive approach to auditory hallucinations. Br J Psychiatry (1994) 164(2):190201. doi: 10.1192/bjp.164.2.190

27. Mawson A, Cohen K, Berry K. Reviewing evidence for the cognitive model of auditory hallucinations: The relationship between cognitive voice appraisals and distress during psychosis. Clin Psychol Rev (2010) 30(2):248-58. doi: 10.1016/j.cpr.2009.11.006

28. Shergill SS, Murray RM, McGuire PK. Auditory hallucinations: a review of psychological treatments. Schizophr Res (1998) 32(2):137-50. doi: 10.1016/ S0920-9964(98)00052-8

29. Brekke JS, Hoe M, Long J, Green MF. How neurocognition and social cognition influence functional change during community-based psychosocial rehabilitation for individuals with schizophrenia. Schizophr Bull (2007) 33(5):1247-56. doi: 10.1093/schbul/sbl072

30. Pilling S, Bebbington P, Kuipers E, Garety P, Geddes J, Orbach G, et al. Psychological treatments in schizophrenia: I. Meta-analysis of family intervention and cognitive behavioural therapy. Psychol Med (2001) 32 (5):763-82. doi: 10.1017/S0033291702005895

31. Pilling S, Bebbington P, Kuipers E, Garety P, Geddes J, Martindale B, et al. Psychological treatments in schizophrenia: II. Meta-analyses of randomized controlled trials of social skills training and cognitive remediation. Psychol Med (2002) 32(5):783-91. doi: 10.1017/ S0033291702005640

32. Tarrier $\mathrm{N}, \mathrm{W}$ ykes $\mathrm{T}$. Is there evidence that cognitive behavioural therapy is an effective treatment for schizophrenia? A cautious or cautionary tale? Behav Res Ther (2004) 42(12):1377-401. doi: 10.1016/j.brat.2004.06.020

33. Ruddle A, Mason O, Wykes T. A review of hearing voices groups: evidence and mechanisms of change. Clin Psychol Rev (2011) 31(5):757-66. doi: 10.1016/j.cpr.2011.03.010

34. Lincoln TM, Peters E. A systematic review and discussion of symptom specific cognitive behavioural approaches to delusions and hallucinations. Schizophr Res (2019) 203:66-79. doi: 10.1016/j.schres.2017.12.014

35. Wykes T, Steel C, Everitt B, Tarrier N. Cognitive behavioural therapy for schizophrenia: effect sizes, clinical models, and methodological rigor. Schizophr Bull (2008) 34(3):523-37. doi: 10.1093/schbul/sbm114

36. Rector NA, Beck AT. Cognitive behavioural therapy for schizophrenia: an empirical review. J Nerv Ment Dis (2001) 189(5):278-87. doi: 10.1097/ 00005053-200105000-00002

37. Sivec HJ, Montesano VL. Cognitive behavioural therapy for psychosis in clinical practice. Psychother (Chic). (2012) 49(2):258-70. doi: 10.1037/a0028256

38. Lynch O, Laws KR, McKenna PJ. Cognitive behavioural therapy for major psychiatric disorder: does it really work? A meta-analytical review of wellcontrolled trials. Psychol Med (2010) 40(1):9-24. doi: 10.1017/ S003329170900590X

39. Pfammatter M, Junghan UM, Brenner HD. Efficacy of psychological therapy in schizophrenia: conclusions from meta-analyses. Schizophr Bull (2006) 32 (1):S64-80. doi: 10.1093/schbul/sbl030

40. van der Gaag M, Valmaggia LR, Smit F. The effects of individually tailored formulation-based cognitive behavioural therapy in auditory hallucinations and delusions: a meta-analysis. Schizophr Res (2014) 156(1):30-7. doi: 10.1016/j.schres.2014.03.016 
41. Zimmermann G, Favrod J, Trieu VH, Pomini V. The effect of cognitive behavioural treatment on the positive symptoms of schizophrenia spectrum disorders: a meta-analysis. Schizophr Res (2005) 77(1):1-9. doi: 10.1016/ j.schres.2005.02.018

42. Sarin F, Wallin L, Widerlov B. Cognitive behavioural therapy for schizophrenia: a meta-analytical review of randomized controlled trials. Nord J Psychiatry (2011) 65(3):162-74. doi: 10.3109/08039488.2011.577188

43. Haddock G, Tarrier N, Spaulding W, Yusupoff L, Kinney C, McCarthy E. Individual cognitive-behaviour therapy in the treatment of hallucinations and delusions: a review. Clin Psychol Rev (1998) 18(7):821-38. doi: 10.1016/ s0272-7358(98)00007-5

44. NICE. Schizophrenia: Core Interventions in the Treatment and Management of Schizophrenia in Primary and Secondary Care (Update). Early Intervention Services. London, England: National Institute of Clinical Excellence (2009).

45. Wykes T, Hayward P, Thomas N, Green N, Surguladze S, Fannon D, et al. What are the effects of group cognitive behavioural therapy for voices? A randomised control trial. Schizophr Res (2005) 77(2-3):201-10. doi: 10.1016/ j.schres.2005.03.013

46. Newton E, Landau S, Smith P, Monks P, Shergill S, Wykes T. Early psychological intervention for auditory hallucinations: An exploratory study of young people's voices groups. J nervous Ment Dis (2005) 193 (1):58-61. doi: 10.1097/01.nmd.0000149220.91667.fa

47. Penn D Meyers P, Evans E, Wirth R, Cai K, Burhinal M. A randomized controlled trial of group cognitive-behavioural therapy vs. enhanced supportive therapy for auditory hallucinations. Schizophr Res (2009) 109 (1-3):52-9. doi: 10.1016/j.schres.2008.12.009

48. McLeod T, Morris M, Birchwood M, Dovey A. Cognitive behavioural therapy group work with voice hearers. Part 1. Br J Nurs (2007) 16 (4):248-52. doi: 10.12968/bjon.2007.16.4.22995

49. McLeod T, Morris M, Birchwood M, Dovey A. Cognitive behavioural therapy group work with voice hearers. Part 2. Br J Nurs (2007) 16 (5):292-5. doi: 10.12968/bjon.2007.16.5.23005

50. Bach $\mathrm{P}$, Hayes $\mathrm{S}$. The use of acceptance and commitment therapy to prevent the rehospitalization of psychotic patients: a randomized controlled trial. J Consult Clin Psychol (2002) 70(5):1129-39. doi: 10.1037/0022006X.70.5.1129

51. Hayes S, Strosahl K, Wilson K. Acceptance and commitment therapy: an experiential approach to behavioural change. New York: Gillford (1992).

52. Gaudiano B, Herbert J. Acute treatment of inpatients with psychotic symptoms using Acceptance and Commitment Therapy: pilot results. Behav Res Ther (2006) 44(3):415-37. doi: 10.1016/j.brat.2005.02.007

53. Wells A. Panic disorder in association with relaxation induced anxiety: An attentional training approach to treatment. Behav Ther (1990) 21(3):273-80. doi: 10.1016/S0005-7894(05)80330-2

54. Carter K, Wells A. Effects of the Attention Training Technique on Auditory Hallucinations in Schizo-Affective Disorder: A Single Case Study. Case Rep Psychiatry (2018) 2018:1-5. doi: 10.1155/2018/1537237

55. Kujala T, Kallio J, Tervaniemi M, Naatanen R. The mismatch negativity as an index of temporal processing in audition. Clin Neurophysiol (2001) 112 (9):1712-9. doi: 10.1016/S1388-2457(01)00625-3

56. Kathmann N, Frodl-Bauch T, Hegerl U. Stability of the mismatch negativity under different stimulus and attention conditions. Clin Neurophysiol (1999) 110(2):317-23. doi: 10.1016/S1388-2457(98)00011-X

57. Sussman E, Winkler I, Wang W. MMN and attention: Competition for deviance detection. Psychophysiol (2003) 40:430-5. doi: 10.1111/14698986.00045

58. Kisley M, Davalos D, Layton H, Pratt D, Ellis J, Seger C. Small changes in temporal deviance modulate mismatch negativity amplitude in humans. Neurosci Lett (2004) 358:194-2000. doi: 10.1016/j.neulet.2004.01.042

59. Davalos D, Kisley M, Freedman R. Behavioural and Electrophysiological Indices of Temporal Processing Dysfunction in Schizophrenia. $J$ Neuropsychiatr Clin Neurosci (2005) 17:517-25. doi: 10.1176/jnp.17.4.517

60. Baldeweg T, Klugman A, Gruzelier J, Hirsch S. Mismatch negativity potentials and cognitive impairment in schizophrenia. Schizophr Res (2004) 69(2-3):203-17. doi: 10.1016/j.schres.2003.09.009

61. Toyomaki A, Kusumi I, Matsuyama T, Kako Y, Ito K, Koyama T. Tone duration mismatch negativity deficits predict impairment of executive function in schizophrenia. Prog Neuropsychopharmacol Biol Psychiatry (2008) 32(1):95-9. doi: 10.1016/j.pnpbp.2007.07.020

62. Kiang M, Light G, Prugh J, Coulson S, Braff D, Kutas M. Cognitive, neurophysiological, and functional correlates of proverb interpretation abnormalities in schizophrenia. J Int Neuropsychol Soc (2007) 13(4):65363. doi: $10.1017 /$ S1355617707070816

63. Kawakubo Y, Kasai K. Support for an association between mismatch negativity and social functioning in schizophrenia. Prog Neuropsychopharmacol Biol Psychiatry (2006) 30(7):1367-8. doi: 10.1016/j.pnpbp.2006.03.003

64. Light G, Braff D. Stability of mismatch negativity deficits and their relationship to functional impairments in chronic schizophrenia. Am J Psychiatry (2005) 162(9):1741-3. doi: 10.1176/appi.ajp.162.9.1741

65. Wynn J, Sugar C, Horan W, Kern R, Green M. Mismatch negativity, social cognition, and functioning in schizophrenia patients. Biol Psychiatry (2010) 67(10):940-7. doi: 10.1016/j.biopsych.2009.11.024

66. Kawakubo Y, Kamio S, Nose T, Iwanami A, Nakagome K, Fukuda M, et al. Phonetic mismatch negativity predicts social skills acquisition in schizophrenia. Psychiatry Res (2007) 152(2-3):261-5. doi: 10.1016/ j.psychres.2006.02.010

67. Light G, Braff D. Mismatch negativity deficits are associated with poor functioning in schizophrenia patients. Arch Gen Psychiatry (2005) 62 (2):127-36. doi: 10.1001/archpsyc.62.2.127

68. Kasai K, Nakagome K, Itoh K, Koshida I, Hata A, Iwanami A, et al. Multiple generators in the auditory automatic discrimination process in humans. Neuroreport (1999) 10(11):2267-71. doi: 10.1097/00001756-199908020-00008

69. Naatanen R, Alho K. Generators of electrical and magnetic mismatch responses in humans. Brain Topogr (1995) 7(4):315-20. doi: 10.1007/ BF01195257

70. Park H, Kwon J, Youn T, Pae J, Kim J, Kim M, et al. Statistical parametric mapping of LORETA using high density EEG and individual MRI: application to mismatch negativities in schizophrenia. Hum Brain Mapp (2002) 17(3):168-78. doi: 10.1002/hbm.10059

71. Schairer K, Gould H, Pousson M. Source generators of mismatch negativity to multiple deviant stimulus types. Brain Topogr. (2001) 14(2):117-30. doi: 10.1023/A:1012992829580

72. Schall U, Catts S, Karayanidi F, Ward P. Auditory event-related potential indices of fronto-temporal information processing in schizophrenia syndromes: valid outcome prediction of clozapine therapy in a three-year follow-up. Int J Neuropsychopharmacol (1999) 2(2):83-93. doi: 10.1017/ S1461145799001418

73. Sato Y, Yabe H, Todd J, Michie P, Shinozaki N, Sutoh T, et al. Impairment in activation of a frontal attention-switch mechanism in schizophrenic patients. Biol Psychol (2003) 62(1):49-63. doi: 10.1016/S0301-0511(02) 00113-8

74. Muller B, Juptner M, Jentzen W, Muller S. Cortical activation to auditory mismatch elicited by frequency deviant and complex novel sounds: a PET study. Neuroimage (2002) 17(1):231-9. doi: 10.1006/ nimg. 2002.1176

75. Alho K, Woods D, Algazi A, Knight R, Naatanen R. Lesions of frontal cortex diminish the auditory mismatch negativity. Electroencephalogr Clin Neurophysiol (1994) 91(5):353-62. doi: 10.1016/0013-4694(94)00173-1

76. Schall U, Johnston P, Todd J, Ward P, Michie P. Functional neuroanatomy of auditory mismatch processing: an event-related fMRI study of durationdeviant oddballs. Neuroimage (2003) 20(2):729-36. doi: 10.1016/S1053-8119 (03)00398-7

77. Taylor M, Perera U. NICE CG178 Psychosis and Schizophrenia in Adults: Treatment and Management - an evidence-based guideline? Br J Psychiatry (2015) 206(5):357-9. doi: 10.1192/bjp.bp.114.155945

78. Kreyenbuhl J, Buchanan R, Dickerson F, Dixon L. The Schizophrenia Patient Outcomes Research Team (PORT): Updated Treatment Recommendations 2009. Schizophr Bull (2010) 36(1):94-103. doi: 10.1093/schbul/sbp130

79. Haddock G, Devane S, Bradshaw T, McGovern J, Tarrier N, Kinderman P, et al. An investigation into the psychometric properties of the Cognitive Therapy Scale for Psychosis (CTS-Psy). Behav Cogn Psychother (2011) 29 (2):221-33. doi: 10.1017/S1352465801002089

80. Haddock G, McCarron J, Tarrier N, Faragher E. Scales to measure dimensions of hallucinations and delusions: the psychotic symptom rating 
scales (PSYRATS). Psychol Med (1999) 29(4):879-89. doi: 10.1017/ S0033291799008661

81. Chadwick P, Lees S, Birchwood M. The revised Beliefs About Voices Questionnaire (BAVQ-R). Br J Psychiatry (2000) 177:229-32. doi: 10.1192/bjp.177.3.229

82. Shawyer F, Ratcliff K, Mackinnon A, Farhall J, Jayes S, Copolov D. The voices acceptance and action scale (VAAS): Pilot data. J Clin Psychol (2007) 63(6):593-606. doi: 10.1002/jclp.20366

83. Greenwood K, Sweeney A, Williams S, Garety P, Kuipers E, Scott J, et al. CHoice of Outcome In Cbt for psychosEs (CHOICE): the development of a new service user-led outcome measure of CBT for psychosis. Schizophr Bull (2010) 36(1):126-35. doi: 10.1093/schbul/sbp117

84. Naatanen R, Pakarinen S, Rinne T, Takegata R. The mismatch negativity (MMN): towards the optimal paradigm. Clin Neuriphysiol. (2004) 115 (1):140-4. doi: 10.1016/j.clinph.2003.04.001

85. Chatrian G, Lettich E, Nelson P. Ten Percent Electrode System for Topographic Studies of Spontaneous and Evoked EEG Activities. Am J EEG Technol (1985) 25:83-92. doi: 10.1080/00029238.1985.11080163

86. Sabri M, Campbell K. The effects of digital filtering on mismatch negativity in wakefulness and slow-wave sleep. J Sleep Res (2002) 11(2):123-7. doi: 10.1046/j.1365-2869.2002.00292.x

87. Graton G, Coles M, Donchin E. A new method for off-line removal of ocular artifact. Electroencephalogr Clin Neurophysiol (1983) 55(4):468-84. doi: 10.1016/0013-4694(83)90135-9

88. Rosburg T, Boutros NN, Ford JM. Reduced auditory evoked potential component N100 in schizophrenia-a critical review. Psychiatry Res (2008) 161(3):259-74. doi: 10.1016/j.psychres.2008.03.017

89. Olbrich S, Mulert C, Karch S, Trenner M, Leicht G, Pogarell O, et al. EEGvigilance and BOLD effect during simultaneous EEG/fMRI measurement. Neuroimage (2009) 45(2):319-32. doi: 10.1016/j.neuroimage.2008.11.014

90. Pascual-Marqui R. Discrete, 3D distributed, linear imaging methods of electric neuronal activity. Part 1: exact, zero error localization. (2007). arXiv:0710.3341 [math-ph]. http://arxiv.org/pdf/0710.3341.

91. Pascual-Marqui R, Lehmann D, Koukkou M, Kochi K, Anderer P, Saletu B, et al. Assessing interactions in the brain with exact low-resolution electromagnetic tomography. Philos Trans A Math Phys Eng Sci (2001) 369(1952):3768-84. doi: 10.1098/rsta.2011.0081

92. Jurcak V, Tsuzuki D, Dan I. 10/20, 10/10, and 10/5 systems revisited: their validity as relative head-surface-based positioning systems. Neuroimage. (2007) 34(4):1600-11. doi: 10.1016/j.neuroimage.2006.09.024

93. Oostenveld R, Praamstra P. The five percent electrode system for highresolution EEG and ERP measurements. Clin Neurophysiol (2001) 112 (4):713-9. doi: 10.1016/S1388-2457(00)00527-7

94. Lancaster J, Woldorff M, Parsons L, Liotti M, Freitas CS, Rainey L, et al. Automated Talairach atlas labels for functional brain mapping. Hum Brain Mapp (2000) 10(3):120-31. doi: 10.1002/1097-0193(200007)10:3<120::AIDHBM30>3.0.CO;2-8

95. Mazziotta J, Toga A, Evans A, Fox P, Lancaster J, Zilles K, et al. A probabilistic atlas and reference system for the human brain: International Consortium for Brain Mapping (ICBM). Philos Trans R Soc Lond B Biol Sci (2001) 356(1412):1293-322. doi: 10.1098/rstb.2001.0915

96. Fuchs M, Kastner J, Wagner M, Hawes S, Ebersole J. A standardized boundary element method volume conductor model. Clin Neurophysiol (2002) 113(5):702-12. doi: 10.1016/S1388-2457(02)00030-5

97. Leicht G, Kirsch V, Giegling I, Karch S, Hantschk I, Möller H-J, et al. Reduced early auditory evoked gamma-band response in patients with schizophrenia. Biol Psychiatry (2010) 67(3):224-31. doi: 10.1016/ j.biopsych.2009.07.033

98. Worrell G, Lagerlund T, Sharbrough F, Brinkmann BH, Busacker NE, Cicora $\mathrm{KM}$, et al. Localization of the epileptic focus by low-resolution electromagnetic tomography in patients with a lesion demonstrated by MRI. Brain Topogr (2000) 12(4):273-82. doi: 10.1023/A:1023407521772

99. Mulert C, Jager L, Schmitt R, Bussfeld P, Pogarell O, Möller H-J, et al. Integration of fMRI and simultaneous EEG: towards a comprehensive understanding of localization and time-course of brain activity in target detection. Neuroimage (2004) 22(1):83-94. doi: 10.1016/j.neuroimage. 2003.10.051
100. Mulert C, Jager L, Propp S, Karch S, Störmann S, Pogarell O, et al. Sound level dependence of the primary auditory cortex: Simultaneous measurement with 61-channel EEG and fMRI. Neuroimage (2005) 28(1):49-58. doi: 10.1016/j.neuroimage.2005.05.041

101. Zumsteg D, Friedman A, Wieser H, Wennberg R. Propagation of interictal discharges in temporal lobe epilepsy: correlation of spatiotemporal mapping with intracranial foramen ovale electrode recordings. Clin Neurophysiol (2006) 117(12):2615-26. doi: 10.1016/j.clinph.2006.07.319

102. Lecomte T, Leclerc C, Corbiere M, Wykes T, Wallace CJ, Spidel A. Group Cognitive behavioural therapy or Social Skills Training for Individuals With a Recent Onset of Psychosis?: Results of a Randomized Controlled Trial. J Nervous Ment Dis (2008) 196(12):866-75. doi: 10.1097/NMD.0b013 e31818ee231

103. Kennedy L, Xyrichis A. Cognitive Behavioural Therapy Compared with Non-specialized Therapy for Alleviating the Effect of Auditory Hallucinations in People with Reoccurring Schizophrenia: A Systematic Review and Meta-analysis. Community Ment Health J (2017) 53(2):12733. doi: 10.1007/s10597-016-0030-6

104. Pontillo M, De Crescenzo F, Vicari S, Pucciarini M, Averna R, Santonastaso $\mathrm{O}$, et al. Cognitive behavioural therapy for auditory hallucinations in schizophrenia: A review. World J Psychiatry (2016) 6(3):372-80. doi: 10.5498/wjp.v6.i3.372

105. Aleman A, Laroi F. Insights into hallucinations in schizophrenia: novel treatment approaches. Expert Rev Neurother. (2011) 11(7):1007-15. doi: 10.1586/ern.11.90

106. Chadwick P, Sambrooke S, Rasch S, Davies E. Challenging the omnipotence of voices: group cognitive behavioural therapy for voices. Behav Res Ther (2000) 38(10):993-1003. doi: 10.1016/S0005-7967(99)00126-6

107. Wong A, Ting K, Chen E. Group cognitive behavioural therapy for Chinese patients with psychotic disorder: A feasibility controlled study. Asian J Psychiatr (2019) 39:157-64. doi: 10.1016/j.ajp.2018.12.015

108. Beck-Sander A, Birchwood M, Chadwick P. Acting on command hallucinations: a cognitive approach. Br J Clin Psychol (1997) 36(Pt 1):139-48. doi: 10.1111/j.2044-8260.1997.tb01237.x

109. Bucci S, Birchwood M, Twist L, Tarrier N, Emsley R, Haddock G. Predicting compliance with command hallucinations: anger, impulsivity and appraisals of voices' power and intent. Schizophr Res (2013) 147(1):163-8. doi: 10.1016/ j.schres.2013.02.037

110. Krakvik B, Stiles T, Hugdahl K. Experiencing malevolent voices is associated with attentional dysfunction in psychotic patients. Scand J Psychol (2013) 54 (2):72-7. doi: 10.1111/sjop.12024

111. Erickson M, Ruffle A, Gold J. A Meta-Analysis of Mismatch Negativity in Schizophrenia: From Clinical Risk to Disease Specificity and Progression. Biol Psychiatry (2016) 79(1):980-7. doi: 10.1016/j.biopsych.2015.08.025

112. Umbricht D, Krljes S. Mismatch negativity in schizophrenia: a meta-analysis. Schizophr Res (2005) 76(1):1-23. doi: 10.1016/j.schres.2004.12.002

113. Fisher D, Labelle A, Knott V. Alterations of mismatch negativity (MMN) in schizophrenia patients with auditory hallucinations experiencing acute exacerbation of illness. Schizpophr Res (2012) 139(1-3):237-45. doi: 10.1016/j.schres.2012.06.004

114. Fisher D, Smith D, Labelle A, Knott V. Attenuation of mismatch negativity (MMN) and novelty P300 in schizophrenia patients with auditory hallucinations experiencing acute exacerbation of illness. Biol Psychol (2014) 100:43-9. doi: 10.1016/j.biopsycho.2014.05.005

115. Javitt DC, Steinschneider M, Schroeder CE, Arezzo JC. Role of cortical Nmethyl-D-aspartate receptors in auditory sensory memory and mismatch negativity generation: implications for schizophrenia. Proc Natl Acad Sci (1996) 93(21):11962-7. doi: 10.1073/pnas.93.21.11962

116. Rosburg T, Kreitschmann-Andermahr I. The effects of ketamine on the mismatch negativity (MMN) in humans-a meta-analysis. Clin Neurophysiol (2016) 127(2):1387-94. doi: 10.1016/j.clinph.2015.10.062

117. De La Salle S, Shah D, Choueiry J, Bowers H, McIntosh J, Ilivitsky V, et al. NMDA Receptor Antagonist Effects on Speech-Related Mismatch Negativity and Its Underlying Oscillatory and Source Activity in Healthy Humans. Front Pharmacol (2019) 10:455. doi: 10.3389/fphar.2019.00455

118. Martin AMS, Bartolomeo L, Howell J, Hetrick WP, Bolbecker AR, Breier A, et al. Auditory feature perception and auditory hallucinatory experiences in 
schizophrenia spectrum disorder. Eur Arch Psychiatry Clin Neurosci (2018) 268(7):653-61. doi: 10.1007/s00406-017-0839-1

119. Dondé C, Silipo G, Dias EC, Javitt DC. Hierarchical deficits in auditory information processing in schizophrenia. Schizophr Res (2019) 206:135-41. doi: 10.1016/j.schres.2018.12.001

120. Plack CJ, Barker D, Hall DA. Pitch coding and pitch processing in the human brain. Hearing Res (2014) 307:53-64. doi: 10.1016/j.heares.2013.07.020

121. De Angelis V, De Martino F, Moerel M, Santoro R, Hausfeld L, Formisano E. Cortical processing of pitch: Model-based encoding and decoding of auditory fMRI responses to real-life sounds. NeuroImage. (2018) 180:291300. doi: 10.1016/j.neuroimage.2017.11.020

122. Alho K, Rinne T, Herron TJ, Woods DL. Stimulus-dependent activations and attention-related modulations in the auditory cortex: a meta-analysis of fMRI studies. Hearing Res (2014) 307:29-41. doi: 10.1016/j.heares.2013.08.001

123. Kompus K, Falkenberg LE, Bless JJ, Johnsen E, Kroken RA, Kråkvik B, et al. The role of the primary auditory cortex in the neural mechanism of auditory verbal hallucinations. Front Hum Neurosci (2013) 7:144. doi: 10.3389/ fnhum.2013.00144

124. Salisbury DF, Kuroki N, Kasai K, Shenton ME, McCarley RW. Progressive and interrelated functional and structural evidence of post-onset brain reduction in schizophrenia. Arch Gen Psychiatry (2007) 64(5):521-9. doi: 10.1001/archpsyc.64.5.521

125. Neckelmann G, Specht K, Lund A, Ersland L, Smievoll AI, Neckelmann D, et al. MR morphometry analysis of grey matter volume reduction in schizophrenia: association with hallucinations. Int J Neurosci (2006) 116 (1):9-23. doi: 10.1080/00207450690962244

126. Hugdahl K, Craven AR, Nygård M, Løberg EM, Berle JØ, Johnsen E, et al. Glutamate as a mediating transmitter for auditory hallucinations in schizophrenia: A 1H MRS study. Schizophr Res (2015) 161(2-3):252-60. doi: 10.1016/j.schres.2014.11.015

127. Curčić-Blake B, Bais L, Sibeijn-Kuiper A, Pijnenborg HM, Knegtering H, Liemburg E, et al. Glutamate in dorsolateral prefrontal cortex and auditory verbal hallucinations in patients with schizophrenia: a 1H MRS study. Prog Neuropsychopharmacol Biol Psychiatry (2017) 78:132-9. doi: 10.1016/ j.pnpbp.2017.05.020

128. Fletcher P, Frith C. Perceiving is believing: a Bayesian approach to explaining the positive symptoms of schizophrenia. Nat Rev Neurosci (2009) 10(1):4858. doi: $10.1038 / \mathrm{nrn} 2536$

129. Nazimek J, Hunter M, Woodruff P. Auditory hallucinations: expectationperception model. Med Hypotheses. (2012) 78(6):802-10. doi: 10.1016/ j.mehy.2012.03.014

130. Schultz W, Dickinson A. Neuronal coding of prediction errors. Annu Rev Neurosci (2000) 23:473-500. doi: 10.1146/annurev.neuro.23.1.473

131. Friston K. A theory of cortical responses. Philos Trans R Soc Lond B Biol Sci (2005) 360(1456):815-36. doi: 10.1098/rstb.2005.1622

132. Garrido M, Kilner J, Stephan K, Friston K. The mismatch negativity: A review of underlying mechanisms. Clin Neurophysiol (2009) 120(3):453-63. doi: 10.1016/j.clinph.2008.11.029

133. Northoff G, Qin P. How can the brain's resting state activity generate hallucinations? A 'resting state hypothesis' of auditory verbal hallucinations. Schizophr Res (2011) 127(1-3):202-14. doi: 10.1016/ j.schres.2010.11.009

134. Northoff G. Are Auditory Hallucinations Related to the Brain's Resting State Activity? A 'Neurophenomenal Resting State Hypothesis'. Clin Psychopharmacol Neurosci (2914) 12(3):189-95. doi: 10.9758/cpn.2014.12.3.189

135. Berlot E, Formisano E, De Martino F. Mapping frequency-specific tone predictions in the human auditory cortex at high spatial resolution. J Neurosci (2018) 38(21):4934-42. doi: 10.1523/JNEUROSCI.2205-17.2018

136. Todd J, Harms L, Schall U, Michie P. Mismatch negativity: translating the potential. Front Psychiatry (2013) 4:171. doi: 10.3389/fpsyt.2013.00171

137. Carbajal GV, Malmierca MS. The neuronal basis of predictive coding along the auditory pathway: From the subcortical roots to cortical deviance detection. Trends Hearing (2018) 22:1-33. doi: 10.1177/2331216518784822

138. Wacongne C. A predictive coding account of MMN reduction in schizophrenia. Biol Psychol (2016) 116:68-74. doi: 10.1016/j.biopsycho.2015.10.011

139. Schmidt A, Diaconescu AO, Kometer M, Friston KJ, Stephan KE, Vollenweider FX. Modeling ketamine effects on synaptic plasticity during the mismatch negativity. Cereb Cortex (2012) 23(10):2394-406. doi: 10.1093/ cercor/bhs 238

140. Baldeweg T. ERP repetition effects and mismatch negativity generation: A predictive coding perspective. J Psychophysiol (2007) 21(3-4):204-13. doi: 10.1027/0269-8803.21.34.204

141. McCleery A, Mathalon D, Wynn J, Roach B, Hellemann G, Marder S, et al. Parsing components of auditory predictive coding in schizophrenia using a roving standard mismatch negativity paradigm. Psychol Med (2019) 49 (7):1195-206. doi: 10.1017/S0033291718004087

142. McCleery A, Wynn J, Mathalon D, Roach B, Green M. Hallucinations, neuroplasticity, and prediction errors in schizophrenia. Scand J Psychol (2018) 59(1):41-8. doi: 10.1111/sjop.12413

143. Linden D. How psychotherapy changes the brain-the contribution of functional neuroimaging. Mol Psychiatry (2006) 11(6):528-38. doi: 10.1038/sj.mp. 4001816

144. Weingarten C, Strauman T. Neuroimaging for psychotherapy research: current trends. Psychother Res (2015) 25(2):185-213. doi: 10.1080/ 10503307.2014.883088

145. Wykes T, Hyddy V, Cellard C, McGurk S, Czobor P. A meta-analysis of cognitive remediation for schizophrenia: methodology and effect sizes. Am J Psychiatry (2011) 168(5):472-85. doi: 10.1176/appi.ajp.2010.10060855

146. Isaac C, Januel D. Neural correlates of cognitive improvements following cognitive remediation in schizophrenia: a systematic review of randomized trials. Socioaffect Neurosci Psychol (2016) 6:30054. doi: 10.3402/snp.v6.30054

147. Perez V, Tarasenko M, Miyakoshi M, Pianka ST, Makeig SD, Braff DL, et al. Mismatch Negativity is a Sensitive and Predictive Biomarker of Perceptual Learning During Auditory Cognitive Training in Schizophrenia. Neuropsychopharmacology (2017) 42(11):2206-13. doi: 10.1038/npp.2017.25

148. Tarasenko M, Perez V, Pianka S, Vinogradov S, Braff DL, Swerdlow NR, et al. Measuring the capacity for auditory system plasticity: An examination of performance gains during initial exposure to auditory-targeted cognitive training in schizophrenia. Schizophr Res (2016) 172(1-3):123-30. doi: 10.1016/j.schres.2016.01.019

149. Thomas M, Bismark A, Joshi Y, Tarasenko M, Treichler E, Hochberger WC, et al. Targeted cognitive training improves auditory and verbal outcomes among treatment refractory schizophrenia patients mandated to residential care. Schizophr Res (2018) 202:378-84. doi: 10.1016/j.schres.2018.07.025

150. Thomas M, Green M, Hellemann G, Sugar CA, Tarasenko M, Calkins ME, et al. Modeling Deficits From Early Auditory Information Processing to Psychosocial Functioning in Schizophrenia. JAMA Psychiatry (2017) 74 (1):37-46. doi: 10.1001/jamapsychiatry.2016.2980

151. Medalia A, Saperstein AM, Qian M, Javitt DC. Impact of baseline early auditory processing on response to cognitive remediation for schizophrenia. Schizophr Res (2019) 208:397-405. doi: 10.1016/j.schres.2019.01.012

152. Kasai K, Nakagome K, Itoh K, Koshida I, Hata A, Iwanami A, et al. Impaired cortical network for preattentive detection of change in speech sounds in schizophrenia: a high-resolution event-related potential study. Am J Psychiatry (2002) 159(4):546-53. doi: 10.1176/appiajp.159.4.546

153. Moerel M, De Martino F, Formisano E. Processing of natural sounds in human auditory cortex: tonotopy, spectral tuning, and relation to voice sensitivity. J Neurosci (2012) 32(41):14205-16. doi: 10.1523/ JNEUROSCI.1388-12.2012

154. Santoro R, Moerel M, De Martino F, Goebel R, Ugurbil K, Yacoub E, et al. Encoding of natural sounds at multiple spectral and temporal resolutions in the human auditory cortex. PloS Comput Biol (2014) 10(1):e1003412. doi: 10.1371/journal.pcbi.1003412

155. Hugdahl K. Auditory Hallucinations as Translational Psychiatry: Evidence from Magnetic Resonance Imaging. Balkan Med J (2017) 34(6):504-13. doi: 10.4274/balkanmedj.2017.1226

156. Hugdahl K, Nygård M, Falkenberg LE, Kompus K, Westerhausen R, Kroken R, et al. Failure of attention focus and cognitive control in schizophrenia patients with auditory verbal hallucinations: evidence from dichotic listening. Schizophr Res (2013) 147(2-3):301-9. doi: 10.1016/j.schres.2013.04.005

157. Hugdahl K, Løberg EM, Falkenberg LE, Johnsen E, Kompus K, Kroken RA, et al. Auditory verbal hallucinations in schizophrenia as aberrant lateralized speech perception: evidence from dichotic listening. Schizophr Res (2012) 140(1-3):59-64. doi: 10.1016/j.schres.2012.06.019 
158. Santoro R, Moerel M, De Martino F, Valente G, Ugurbil K, Yacoub E, et al. Reconstructing the spectrotemporal modulations of real-life sounds from fMRI response patterns. Proc Natl Acad Sci (2017) 114(18):4799-804. doi: 10.1073/pnas.1617622114

159. Dunn G, Emsley R, Liu H, Landau S. Integrating biomarker information within trials to evaluate treatment mechanisms and efficacy for personalised medicine. Clin Trials (2013) 10(5):709-19. doi: 10.1177/ 1740774513499651

160. Sommer IE, Kleijer H, Hugdahl K. Toward personalized treatment of hallucinations. Curr Opin Psychiatry (2018) 31(3):237-45. doi: 10.1097/ YCO.0000000000000416
Conflict of Interest: The authors declare that the research was conducted in the absence of any commercial or financial relationships that could be construed as a potential conflict of interest.

Copyright (C) 2020 Knott, Wright, Shah, Baddeley, Bowers, de la Salle and Labelle. This is an open-access article distributed under the terms of the Creative Commons Attribution License (CC BY). The use, distribution or reproduction in other forums is permitted, provided the original author(s) and the copyright owner(s) are credited and that the original publication in this journal is cited, in accordance with accepted academic practice. No use, distribution or reproduction is permitted which does not comply with these terms. 